\title{
Rursus
}

Russus

Poiétique, réception et réécriture des textes antiques

$8 \mid 2012$

Les épitomés scientifiques et historiques

\section{Du texte d'origine à l'Épitomé des Ethnika: Les différentes phases de réduction et la transmission du lexique géographique de Stéphane de Byzance}

From the geographical lexicon of Stephanus of Byzantium to the Ethnika's

Epitome. Various stages of reduction and transmission of the text

\section{Marc Bouiron}

\section{OpenEdition}

Journals

Édition électronique

URL : http://journals.openedition.org/rursus/1027

DOI : $10.4000 /$ rursus. 1027

ISSN : 1951-669X

Éditeur

Université Nice-Sophia Antipolis

Édition imprimée

Date de publication : 14 décembre 2012

Référence électronique

Marc Bouiron, « Du texte d'origine à l'Épitomé des Ethnika : Les différentes phases de réduction et la transmission du lexique géographique de Stéphane de Byzance », Rursus [En ligne], 8 | 2012, mis en ligne le 29 novembre 2012, consulté le 30 avril 2019. URL : http://journals.openedition.org/ rursus/1027; DOI : 10.4000/rursus. 1027

Ce document a été généré automatiquement le 30 avril 2019

Rursus 


\section{Du texte d'origine à l'Épitomé des Ethnika: Les différentes phases de réduction et la transmission $\mathrm{du}$ lexique géographique de Stéphane de Byzance}

From the geographical lexicon of Stephanus of Byzantium to the Ethnika's

Epitome. Various stages of reduction and transmission of the text

Marc Bouiron

\section{Introduction}

1 Stéphane de Byzance est probablement un des auteurs les plus cités par les historiens et archéologues travaillant sur l'histoire des lieux antiques. Son ouvrage a en effet préservé d'une disparition totale de très nombreuses mentions d'auteurs anciens, principalement de géographes et d'historiens, se rapportant à des toponymes. Depuis la Renaissance et l' editio princeps d'Aldus, en 1502, les éditions ont été nombreuses; chacune a apporté une pierre critique nécessaire à une meilleure compréhension du texte d'origine ${ }^{1}$.

2 Mais on cherchera en vain dans ces ouvrages une réflexion détaillée sur l'œuvre ellemême. Le seul travail d'envergure sur cette œuvre reste l'article qu'a rédigé Ernst Honigmann dans la Real-Encyclopädie de Pauly-Wissowa en 1929. Quasiment tous les arguments - même récents - concernant la datation de l'œuvre, le découpage en livres ou les sources utilisées par Stéphane de Byzance sont issus de cette étude qui reprend de nombreuses réflexions des différents éditeurs, ses prédécesseurs, en les approfondissant. Mais ce travail, pourtant le plus achevé de toutes les études sur Stéphane de Byzance, n'est pas assez complet et ne permet pas en particulier de comprendre les différentes phases d'abréviation. 
Il est difficile de réfléchir à la valeur historique des notices des Ethnika sans tenir compte de cette phase de réécriture. L'analyse peut alors mener à des conclusions inexactes ou approximatives. C'est pourquoi il nous a semblé primordial de consacrer une large part de notre réflexion ${ }^{2}$ au travail d'abréviation. Le fait de n'avoir conservé qu'un abrégé (un résumé? une paraphrase?) implique d'abord d'avoir identifié le processus de transformation de l'œuvre originale. L'analyse de l'Épitomé et des quelques fragments subsistant de l'œuvre d'origine permettent de mieux comprendre comment Stéphane de Byzance a élaboré son ouvrage et quelle était son étendue réelle. Ceci passe par la comparaison systématique des notices conservées et par la compréhension de la structure du lexique à la fois dans l'œuvre originale et dans l'Épitomé.

\section{Prolégomènes : l'ouvrage initial et sa datation}

\subsection{L'auteur et le titre}

4 Nous possédons encore un fragment de l'œuvre d'origine dans quelques feuillets du manuscrit Parisinus Coislinianus 228 (S), datés $\mathrm{du} \mathrm{XI}^{\mathrm{e}}$ siècle ${ }^{3}$. Les quelques folios conservés nous ont préservé la fin d'un livre (le XIII) avec les notices comprises entre Dumânes ${ }^{4}$ et Dôtion, ainsi qu'une table des matières (pinax) du livre suivant (une partie de la lettre $\varepsilon^{5}$ ) et le début de la première notice, celle de Eares. Le livre XIV débute (fol. 122) par un en-tête

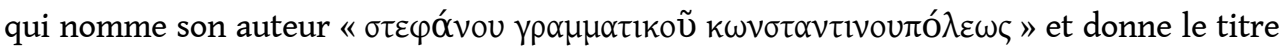
de l'ouvrage. Nous aurons l'occasion à plusieurs reprises de revenir sur ce fragment, d'une importance capitale pour la compréhension de l'œuvre d'origine et son abrégé.

Plus tard, au milieu du x siècle, l'auteur ${ }^{6}$ du livre II du De Thematibus $(10,9)$ l'appelait « $\dot{0}$

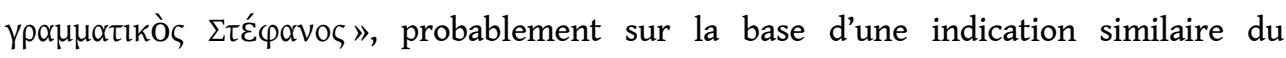
manuscrit qu'il utilisait. Enfin, au xII siècle, Jean Tzetzès (Chiliades III, 818-820)

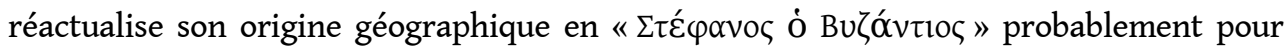
des questions métriques (les Chiliades sont en vers). Ce grammairien n'est connu que par ce seul ouvrage à caractère géographique ${ }^{7}$.

6 L'œuvre d'origine s'appelait vraisemblablement « Au sujet des villes et des îles, des peuples et des dèmes, et des lieux; et, les concernant, des cas d'homonymie et de changement de nom, ainsi que des ethnonymes et des toponymes qui en sont dérivés, et des noms des ktétiques (issus de ces

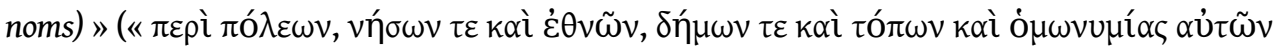

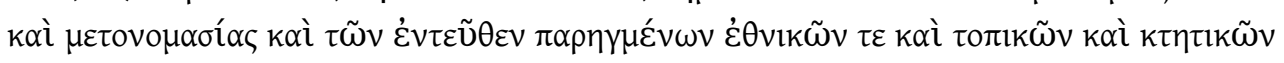
óvouó $\tau \omega v »)$. Ce titre apparaît dans l'en-tête du livre XIV du manuscrit Coislinianus 228, après le nom de l'auteur.

7 Le titre est déjà donné en abrégé (les Ethniques) au début du IX siècle chez le grammairien

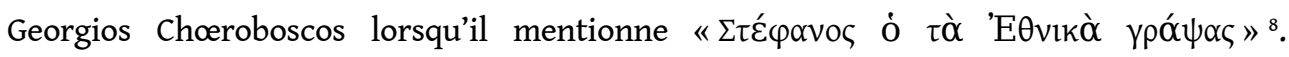
Trois siècles plus tard, Stéphane de Byzance est dénommé l'«auteur d'Ethniques» (EӨviкоүрó $\varphi$ ov) dans les nombreuses mentions d'Eustathe de Thessalonique`, qui ne le

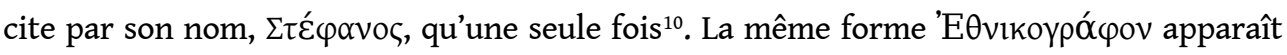
dans les gloses tardives de la Souda (notice Nakônê $\hat{e}^{11}$ ), dont A. DILLER (1938: 335-336) a montré qu'elles étaient de la main du même Eustathe. Il est quoiqu'il en soit intéressant de constater que les auteurs postérieurs au $\mathrm{VI}^{\mathrm{e}}$ siècle ont considéré que son œuvre traitait principalement des noms de peuples et ont fait de cette indication le titre usuel dérivé, peut-être du fait d'une introduction générale maintenant disparue. 


\subsection{La datation}

8 L'œuvre que nous connaissons actuellement semble avoir été composée dans le second quart $\mathrm{du} \mathrm{VI}^{\mathrm{e}}$ siècle de notre ère, comme en témoignent les mentions d'Eugène, grammairien sous Anastase $\mathrm{I}^{\mathrm{er}}(491-518)^{12}$, des nouveaux noms d'Antioche et de Sykai donnés par Justinien après $528^{13}$, ou de la fortification de Dara/Anastasiopolis, construite en 507 et détruite en $573^{14}$. Dernier argument chronologique, il a été proposé d'identifier,

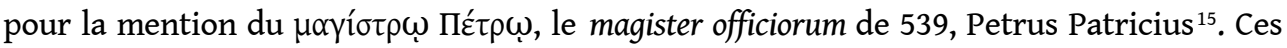
arguments sont connus depuis longtemps et ont été repris et détaillés par E. Honigmann, auquel nous renvoyons ${ }^{16}$. Ce dernier considère que ces mentions sont l'œuvre de Stéphane de Byzance. Nous pouvons ajouter un dernier et nouvel argument : la mention $\mathrm{du}$ « remarquable sophiste » Diogène (rattaché à la mention de sa ville de naissance, Abila ), qui fait partie des philosophes réfugié en Perse à la fermeture de l'école d'Athènes par Justinien en $529^{17}$.

9 L'incertitude sur la datation vient d'un article de la Souda, sous l'entrée 'Eppó̉ coc. Le texte indique qu'un certain Hermolaos, grammatikos, est l'auteur d'un abrégé des Ethnika de Stéphane de Byzance et que cet épitomé avait été dédié à Justinien ${ }^{18}$. Si la dédicace concerne bien l'abrégé comme l'indique la notice, on peut se demander s'il s'agit de Justinien $\mathrm{I}^{\text {er }}$ (empereur de 527 à 565) ou Justinien II (empereur de 685 à 695 et de 705 à 711). Comme A. DILLER (1938, p. 333) en son temps, M. BILLERBECK et alii $\left(2006: 4^{*}\right.$, n. 4) rappelle que la Souda reprend les notices biographiques de l'Onomatologos d'Hesychios de Milet, auteur du $\mathrm{VI}^{\mathrm{e}}$ siècle $^{19}$. Ce dernier pourrait donc être la source de la mention de la dédicace ce qui exclurait alors Justinien II mais pose bien évidemment problème s'il s'agit d'une abréviation d'une œuvre déjà existante. Ceci implique alors qu'Hermolaos était un quasi contemporain de Stéphane de Byzance (DILLER, 1938: 333, n.2) ou bien que ce dernier est nettement antérieur.

10 A. MEINEKE, dans son édition de 1849, pensait que la dédicace concernait bien l'épitomé et plaçait les gloses se rapportant au $\mathrm{VI}^{\mathrm{e}}$ siècle sous la plume d'Hermolaos. Il était pour lui vraisemblable de dater l'œuvre originale du siècle précédent, ce qui lui semblait compatible avec les auteurs cités qui, toujours selon son avis, ne dépassaient pas le début du ve siècle. Pour A. DILLER (1938 : 333, n. 2), la dédicace à Justinien pouvait se rapporter à Stéphane de Byzance lui-même ${ }^{20}$.

11 La nouvelle édition en cours (BILLERBECK et alii, 2006) n'apporte pas d'autres arguments sur la chronologie des deux auteurs. Nous pensons toutefois que l'on peut renforcer l'argumentation d'une datation justinienne pour l'œuvre d'origine en analysant l'un des auteurs cité par Stéphane de Byzance: Capiton de Lycie. Jusqu'ici, on n'a pas suffisamment prêté attention aux citations de cet auteur, le plus tardif utilisé par Stéphane de Byzance. Pour E. HONIGMANN (1929: col. 2386), il s'agit d'un auteur mal daté. Nous savons par la Souda ${ }^{21}$ qu'il écrivit une histoire de l'Isaurie ${ }^{22}$ (Isaurika) en 8 livres $^{23}$ et traduisit en grec l'épitomé de Tite-Live par Eutrope ${ }^{24}$. Dans le texte qui nous est parvenu, Stéphane de Byzance le cite à quinze reprises, dont dix fois explicitement pour son œuvre Isaurika (trois citations pour le livre I, trois pour le livre II, une pour le livre III, une pour le livre $\mathrm{V}$ et deux pour le livre VI) et cinq fois sans mention de titre (mais la localisation géographique des toponymes renvoie très vraisemblablement à la même œuvre). 
Les mentions de Capiton de Lycie ne sont pas différentes de celles des autres auteurs: souvent elles constituent l'unique référence d'un nom; d'autres fois il est clair que Stéphane de Byzance a utilisé d'autres auteurs, que l'abrégé a supprimé. Il n'y a pas de preuve pour y voir une adjonction spécifique d'Hermolaos. Il est probable que l'œuvre de Capiton, qui mentionne le général Conon (mort en 493), s'inscrit dans la série de commémorations de la victoire d'Anastase sur les Isauriens et date des alentours de 500.

\subsection{L'abréviation d'Hermolaos} résoud pas le problème d'Hermolaos. Celui-ci a vécu entre 540 et la rédaction de l'article de la Souda (vers l'extrême fin du $\mathrm{x}^{\mathrm{e}}$ siècle ou au tout début du siècle suivant). Le travail d'Hermolaos, personnage totalement inconnu par ailleurs, a été jugé suffisamment important pour justifier une entrée dans la Souda, alors même que Stéphane de Byzance n'apparaît pas. Tout repose donc sur l'analyse de la Souda, dont on peut signaler par ailleurs qu'elle n'utilise aucune des notices de Stéphane de Byzance et ignore donc l'abréviation d'Hermolaos.

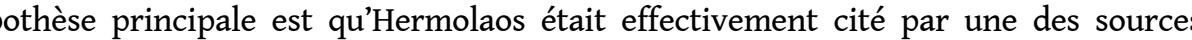
"anciennes» de la Souda. On a remarqué que les notices sur des personnages présentaient une certaine homogénéité dans leur composition, ce qui les a fait attribuer à la seule œuvre mentionnée dans la Souda comme source vraisemblable, l'Onomatologos d'Hésychios de Milet $^{25}$, comme on l'a dit plus haut. Cet auteur, dont la Souda précise qu'il vécut sous le règne de l'empereur Anastase (491-518), a également écrit deux ouvrages historiques dont un sur le règne de Justinien. Il serait donc un quasi-contemporain de Stéphane de Byzance. Enfin, il faut noter que, dans la Souda, toutes les notices de personnages se présentent de la même manière, y compris celle d'Hésychios (alors que ce dernier ne devait probablement pas parler de lui-même ${ }^{26}$.

Il reste toutefois une seconde hypothèse, celle de la présence de la notice d'Hermolaos dans une version révisée d'Hesychios de Milet, avec une mauvaise compréhension du rôle d'Hermolaos qui ne serait pas le dédicataire de l'œuvre mais seulement l'abréviateur. On a pu mettre en évidence, dans la Souda, la présence de biographies jusqu'aux environs du milieu du IX ${ }^{\mathrm{e}}$ siècle. On a donc pensé à l'existence d'un épitomé réactualisé d'Hésychios ${ }^{27}$, auquel s'appliquerait l'indication dans la Souda qu'il s'agit de la source de ce «livre » (comprendre cet ouvrage); ce serait en fait le résumé de l'Onomatologos. Nous avons conservé une ultime version de cet épitomé, sous une forme très réduite: le De viris illustribus du Pseudo-Hesychios, publié par M. MARCovicH (1999-2002 : t. 2, 89-138), dont le texte proche de celui de la Souda rend très vraisemblable l'épitomisation. Celle-ci aurait porté à la fois sur la contraction du texte et le choix de certaines notices puisque le pseudo-Hesychios comporte beaucoup moins de notices que la Souda.

17 Le fait que la Souda n'utilise jamais Stéphane de Byzance ou son abrégé (alors que Constantin Porphyrogénète s'est servi du texte original à plusieurs reprises comme nous le verrons plus loin) est toutefois révélateur. Cela implique qu'Hermolaos n'est pas un contemporain de la Souda et en est bien éloigné. Si l'hypothèse d'un ajout postérieur au VI ${ }^{\mathrm{e}}$ siècle reste théoriquement possible, aucun argument ne vient l'étayer et elle nous 
semble peu probable. Il faut noter que, parmi les grammatikoi signalés par la Souda, Hermolaos apparaît comme le plus récent.

Nous voici donc avec deux auteurs quasiment contemporains, dont l'un a rédigé l'abrégé du second. On peut se demander si Hermolaos n'est pas le successeur de Stéphane, qui aurait en fait édité une partie de son texte plutôt que réellement écrit un abrégé total de l'œuvre. Hermolaos a pu tirer de l'œuvre de Stéphane de Byzance (monumentale comme nous le verrons plus loin et probablement réservée à l'usage des grammairiens) une partie des notices et en faire une œuvre spécifique. C'est elle qu'aurait connu Hesychios ; elle aurait été effectivement dédiée à Justinien, on l'imagine après la mort de Stéphane de Byzance. Or, rien ne nous a été conservé de ce premier abrégé, qui n'est cité par aucun auteur postérieur. Nous admettrons donc (et le reste de l'étude de l'histoire du texte va dans ce sens) que le texte que nous avons conservé n'est pas celui d'Hermolaos mais bien celui de Stéphane de Byzance ; notre Épitomé provient d'une abréviation postérieure. Ceci renouvelle évidemment la vision que nous avons de l'histoire du texte, comme nous pourrons le constater dans la troisième partie de cet article.

\subsection{Le fragment de manuscrit de Stéphane de Byzance} attribué à Stéphane de Byzance. Connu depuis le XVII siècle, il a déjà été publié de façon spécifique à de nombreuses reprises : en 1669 par Samuel Tennelius (= Ten Nuyl), en 1681 par Jacobus Gronovius et en 1715 par Bernard de Montfaucon, pour ne citer que les érudits antérieurs au XIX ${ }^{e}$ siècle. Après avoir décrit le manuscrit, nous analyserons en détail le texte conservé.

Le manuscrit a appartenu d'abord à Pierre Séguier (1588-1672), chancelier et grand amateur de manuscrits grecs qui n'a eu de cesse d'agrandir sa collection. À sa mort, sa bibliothèque est léguée à son arrière petit-fils Henri-Charles du Cambout, duc de Coislin et évêque de Metz (1665-1732) ${ }^{28}$.

Il s'agit d'un codex composite de $283 \times 215 \mathrm{~mm}$ et de 124 folios (dont un folio 18 bis et 109 bis). Il est constitué de plusieurs manuscrits, soit en parchemin et datés du xi siècle (fol. 12-74, 88-95, 116-122), soit en papier d'époques différentes. Le texte de Stéphane de Byzance occupe les folios 116-122v, huit feuillets arrachés d'un manuscrit du $\mathrm{XI}^{\mathrm{e}}$ siècle, à 26 lignes. Le folio 119 bis est lacéré sur toute sa longueur et quasiment illisible. Tous les feuillets ont été rongés sur les bords exérieurs et attaqués par les champignons, ce qui les distingue du reste du manuscrit ${ }^{29}$.

Dans ce manuscrit, le texte de Stéphane de Byzance présente quelques corrections de secondes mains supra lineam ${ }^{30}$ qui sont signalées pour la plupart dans l'apparat critique de l'édition de M. Billerbeck. On peut les ranger en deux catégories: celles qui relèvent certainement d'une vérification du manuscrit d'origine au moment de la copie et celles qui sont nettement postérieures. Il s'agit de lettres ajoutées au-dessus des mots ou de mots complétés sur la ligne ; on note une seule correction d'accent. Dans la très grande majorité des cas il s'agit, de façon classique, de corrections faites à la suite d'une relecture de la copie qui vient d'être réalisée ; le reste correspond à une correction plus tardive, en particulier après disparition de quelques lettres (voir par exemple $\delta 146,58$ ) du fait de déchirures du bord du folio. 
Il faut noter la présence de notes marginales qui n'apparaissent pas dans les éditions du texte et n'ont jamais été signalées. Elles sont placées systématiquement dans la marge extérieure $d u$ folio. On en repère deux séries différentes mais qui semblent être contemporaines ${ }^{31}$ :

- la première est appelée dans le texte par un signe distinctif (un tilde avec un point audessus), avec un commentaire dans la marge extérieure : par exemple au fol. 116v, la note se rapporte au mot vouí̧ovøl (il ne subsiste que quelques lettres ne permettant pas de lire le texte).

- la seconde série commence par l'abréviation de őtı et peut être placée dans la marge extérieure comme dans une ligne située en bas de page. On trouve ainsi peut-être au fol. 116v la citation exacte de Strabon (VII, 5, 8) que le texte donne tronquée; au fol. 117, la mention peut-être de la région d'Illyrie.

$\mathrm{Au}$ folio $117 \mathrm{v}$, en bas de page, l'une des mentions comporte à la fois le signe distinctif et l'abréviation. Le tout est d'une graphie similaire, ce qui n'autorise tout au plus à classer ces différentes annotations qu'en fonction de la nature de la note mais pas de les attribuer à des auteurs différents. Au final, seules les notices de Durrachion, Dôdônê et Doros comportent des annotations. Elles témoignent d'une lecture et d'une utilisation du texte postérieurement à sa rédaction.

L'analyse des erreurs du manuscrit est intéressante car elle permet d'approcher le modèle sur lequel se faisait la copie. Parmi les différentes erreurs, on note le grand nombre de fautes d'onciales liées à une copie d'un texte ancien. Dans le manuscrit $\mathrm{S}$, on trouve tout d'abord de nombreuses fautes d'accentuation ${ }^{32}$; elles témoignent de la façon dont le moine Théophile a restitué l'accentuation sur la base d'un manuscrit qui n'en comportait probablement pas. Les fautes d'onciales plus «classiques " (mécoupures, confusions de lettres...) sont également très nombreuses ${ }^{33}$.

L'ensemble de ces observations témoignent, pour ce reste du manuscrit, d'une copie prise sur un manuscrit en onciales, ce qui implique une certaine ancienneté du modèle. Il s'agit bien d'un fragment du manuscrit de Stéphane de Byzance. Cette détermination est là encore fondamentale pour l'histoire du texte.

\section{Le processus de réduction du texte}

27 Avant d'analyser en détail l'histoire du texte, il nous faut étudier plus finement l'Épitomé qui est parvenu jusqu'à nous en le comparant avec le texte de Stéphane de Byzance. Ce travail n'a jamais été réalisé de manière précise, c'est pourquoi il nous a semblé important de comprendre de façon objective et quantifiée le travail d'abréviation.

\subsection{Prouver la réduction du texte}

Pour comprendre le phénomène de la réduction du texte, il nous faut aborder les preuves objectives de l'abréviation ainsi que les aspects méthodologiques liés à la compréhension de cette abréviation, qui sous-tendent la quantification que nous élaborerons ensuite. 


\subsubsection{Les preuves directes de la réduction}

Comment pouvons-nous être sûrs que le texte a bien connu une ou plusieurs abréviations? Nous disposons heureusement d'éléments parfaitement objectifs.

Le premier d'entre eux est bien évidemment le fait qu'il existe encore un fragment de ce que l'on considèrera comme une copie du manuscrit d'origine. La comparaison avec l' Épitomé prouve de façon simple l'existence d'une abréviation drastique du texte d'origine. Nous reviendrons ci-dessous plus en détail sur la quantification (en nombre et en pourcentage de caractères et de citations) de cette réduction mais nous pouvons déjà constater que nous avons affaire à deux niveaux de texte.

31 Le second indice est constitué d'une double mention de notices, pour le début de la lettre $\chi$ : le manuscrit Rehdigeranus $47^{34}$, qui fait partie des manuscrits majeurs ayant servi à l'édition du texte de l'Épitomé, donne en effet une suite de neuf notices réduites correspondant aux neuf premiers lemmes de la lettre. Les éditeurs (MEINEKE 1849: 676, app. crit. ; WESTERMANN, 1839 : VIII) y ont vu, bien évidemment, la preuve de deux phases d'abréviation. Mais il faut bien noter, même sans étude approfondie, que le texte le moins abrégé est loin d'avoir la longueur du texte d'origine. En particulier, la notice de la ville natale de Plutarque, Chairôneia (MEINEKE, 678 : 1-17), aurait dû comporter cette mention et être largement plus développée. Même si à ce niveau de l'étude il n'est pas possible de déterminer la nature des trois niveaux de texte (celui d'origine, le texte le moins abrégé que l'on trouve dans la plupart des manuscrit et l'abrégé plus important du manuscrit Rehdigeranus 47), ceux-ci semblent manifestes.

Le dernier indice, plus difficile à mettre en évidence ${ }^{35}$, concerne les mentions qu'Eustathe de Thessalonique fait des Ethnika de Stéphane de Byzance : le texte qu'il utilise est dans l'ensemble un peu plus développé que celui que nous connaissons aujourd'hui. Or, il indique avoir lui-même utilisé un épitomé de l'œuvre. Nous avons donc là encore au moins trois niveaux de texte : celui d'origine, l'épitomé qu'utilise Eustathe et le texte que nous avons conservé.

33 La question majeure à laquelle il faut répondre si l'on veut comprendre les différents niveaux d'abréviation est : existe-t-il une correspondance entre les trois niveaux de textes (et donc les deux phases d'abréviation) que l'on peut mettre en évidence ? Ou bien faut-il imaginer plus de phases d'abréviation?

\subsubsection{Les aspects méthodologiques}

Pour répondre à la question du nombre d'abréviations différentes, nous allons dans un premier temps comparer le texte d'origine avec celui de notre Épitomé. On peut considérer en effet que, s'agissant d'un même livre (le XIII), les dernières notices de la lettre $\Delta$ dans l'Épitomé correspondent à une même phase d'abréviation. Nous nous attacherons donc à quantifier la réduction pour aboutir à un pourcentage moyen de conservation du texte (correspondant au complémentaire, mathématiquement parlant, du pourcentage de réduction). Nous considérons que ces notices, étudiées au hasard de la conservation des textes, peuvent être représentatives de l'ensemble des notices: on trouve en effet à la fois des notices de toponymes célèbres (comme Dôdônê ou Dyrrachion) et d'autres attestés par peu d'auteurs (comme Doros) voire un seul auteur (comme Durbaioi ). 
Nous rajouterons à ce corpus les quelques mentions du texte d'origine que l'on trouve dans l'œuvre de Constantin Porphyrogénète. Le texte que réutilise l'empereur n'est que partiel, comme le prouve la notice de Durrachion. Nous tiendrons compte cependant de ces extraits puisqu'ils nous donnent accès au texte d'origine mais ne pourrons pas nous baser sur ces textes pour obtenir les mêmes certitudes sur les différentes abréviations.

Dans un second temps, il sera important de discriminer les différentes phases d'abréviation. Pour cela, nous allons établir une valeur moyenne de la longueur des notices pour l'ensemble des notices de l'Épitomé. Ce chiffre n'a bien sûr qu'une signification théorique, mais il nous permettra d'examiner si, pour un groupe de notices suffisamment important, la longueur moyenne se situe nettement au-dessus ou nettement au-dessous. Nous raisonnerons dans un premier temps par lettre, puis selon la délimitation des livres d'origine, découpage qui nous semble préférable. Ceci nous amènera à reprendre la question du nombre de livre et de leur ampleur, tout en abordant la question des lacunes dans le texte de l'Épitomé.

Enfin, en couplant les différents résultats auxquels nous arriverons, il sera possible d'approcher l'ampleur de l'ouvrage d'origine: la mise en évidence des phases de réduction, pouvant correspondre à des pourcentages de conservation du texte quantifiables, permettront de façon complémentaire de retrouver un nombre approximatif de caractères du manuscrit d'origine.

\subsection{La réduction du texte : aspects quantitatifs}

Nous l'avons dit, quelques citations d'auteurs byzantins et un fragment conservé permettent de se faire une idée de l'œuvre originelle, et surtout de la façon dont elle a été abrégé. Des vingt-deux notices de Stéphane de Byzance qui nous ont été soit totalement soit partiellement conservées, nous avons principalement utilisé pour notre analyse celles comprises entre $\Delta u \mu \tilde{\alpha} v \varepsilon \zeta$ et "E $\alpha \rho \varepsilon \zeta^{36}$, dont on est sûr qu'elles sont intégralement de notre auteur. Nous avons également pris en compte, mais dans une moindre mesure, les mentions de Constantin Porphyrogénète qui correspondent à des parties de notices ${ }^{37}$. En effet, comme nous allons le voir, les citations issues de Constantin sont partielles; pour la plupart, elles ne donnent pas le détail des variantes du nom qui constituent la seconde partie des notices. Nous considérerons, dans un premier temps, que l'abréviation est homogène, avant d'approfondir la question d'abréviations multiples.

\subsubsection{Le facteur d'abréviation}

Afin de mieux appréhender les problèmes liés à l'abréviation du texte de Stéphane de Byzance par l'auteur de l'abrégé, nous avons établi un tableau récapitulatif des notices analysées (tab. I), qui a servi de base pour l'élaboration d'un certain nombre de graphiques.

\begin{tabular}{|l|l|l|l|l|l|l|l|}
\hline \multicolumn{2}{|l|}{} & $\begin{array}{l}\text { Nb de car. } \\
\text { S. de de } \\
\text { Byzance }\end{array}$ & $\begin{array}{l}\text { Nbar. Épitomé } \\
\text { car de consv. }\end{array}$ & $\begin{array}{l}\text { Nb de } \\
\text { du texte } \\
\text { citations } \\
\text { S. de de } \\
\text { Byzance }\end{array}$ & $\begin{array}{l}\text { Nb de } \\
\text { citations } \\
\text { Épitomé }\end{array}$ & $\begin{array}{l}\text { Facteur } \\
\text { de } \\
\text { réduction }\end{array}$ \\
\hline 1 & $\Delta \hat{\prime} \mu \eta$ & 1168 & 123 & $10,5 \%$ & 13 & 1 & 13 \\
\hline
\end{tabular}




\begin{tabular}{|c|c|c|c|c|c|c|c|}
\hline 2 & $\Delta u ́ v \delta \alpha \sigma o v$ & 114 & 40 & $35,1 \%$ & 1 & 0 & \\
\hline 3 & $\Delta v \rho \beta \alpha \tilde{I} 01$ & 439 & 211 & $48,1 \%$ & 1 & 1 & 1 \\
\hline 4 & $\Delta$ uppóxiov & 1585 & 446 & $28,1 \%$ & 10 & 2 & 5 \\
\hline 5 & $\Delta v \sigma \pi$ óv $\tau i o v$ & 417 & 84 & $20,1 \%$ & 3 & 0 & \\
\hline 6 & 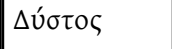 & 134 & 73 & $54,5 \%$ & 1 & 1 & 1 \\
\hline 7 & $\Delta \omega \delta \omega \dot{v} \eta$ & 3693 & 581 & $15,7 \%$ & 25 & 2 & 12,5 \\
\hline 8 & $\Delta \omega v \varepsilon \tau \tau \tilde{I} v 01$ & 129 & 85 & $65,9 \%$ & 2 & 1 & 2 \\
\hline 9 & $\Delta \tilde{\omega} \rho \alpha$ & 257 & 101 & $39,3 \%$ & 2 & 1 & 2 \\
\hline 10 & 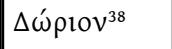 & $>2157$ & 173 & $<8 \%$ & $>9$ & 2 & $>4,5$ \\
\hline 11 & $\Delta \tilde{\omega} \rho \circ \varsigma$ & 1665 & 107 & $6,4 \%$ & 13 & 2 & 6,5 \\
\hline 12 & $\Delta \omega \dot{\tau} \tau 10 v$ & 1392 & 196 & $14,1 \%$ & 15 & 2 & 7,5 \\
\hline 13 & ${ }^{\prime} E \alpha \rho \varepsilon \varsigma$ & 116 & 53 & $45,7 \%$ & 1 & 0 & \\
\hline & moyenne & 1020 & 175 & $17,13 \%$ & 7,38 & 1,15 & non signif. \\
\hline
\end{tabular}

Tab. I. Tableau des notices conservées de Stéphane de Byzance.

Sont ainsi notés le nombre de caractères ${ }^{39}$ des notices originelles, celui des notices abrégées dans l'Épitomé et le pourcentage de conservation du texte (qui correspond mathématiquement parlant à l'inverse du facteur de réduction). La partie droite du tableau donne le nombre de citations présentes chez nos deux auteurs, avec, à titre indicatif, le facteur de réduction. La moyenne qui est donnée en fin de tableau est arrondie à l'entier le plus proche pour le nombre de caractères et au centième pour le reste.

41 Le second tableau (tab. II) correspond aux notices incomplètes, pour lesquelles nous avons calculé les mêmes éléments mais en tenant compte du fait que le nombre de caractères ou de citations sont inférieurs à ceux de la notice d'origine.

\begin{tabular}{|c|c|c|c|c|c|c|c|}
\hline & & $\begin{array}{l}\text { Nb de car. } \\
\text { S. de } \\
\text { Byzance }\end{array}$ & $\begin{array}{l}\mathrm{Nb} \text { de } \\
\text { car. } \\
\text { Épitomé }\end{array}$ & $\begin{array}{l}\text { \% de consv. } \\
\text { du texte }\end{array}$ & $\begin{array}{l}\mathrm{Nb} \text { de } \\
\text { citations } \\
\text { S. de } \\
\text { Byzance }\end{array}$ & $\begin{array}{l}\mathrm{Nb} \text { de } \\
\text { citations } \\
\text { Épitomé }\end{array}$ & $\begin{array}{l}\text { Facteur } \\
\text { de } \\
\text { réduction }\end{array}$ \\
\hline 14 & 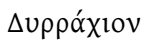 & $>457$ & 318 & $<69,6 \%$ & $>3$ & 1 & $>3$ \\
\hline 15 & 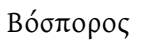 & > 1920 & 820 & $<42,7 \%$ & $>7$ & 3 & $>2,33$ \\
\hline 16 & 'I $\beta \eta \rho i ́ \alpha$ & $>2338$ & 287 & $<12,3 \%$ & $>16$ & 1 & $>16$ \\
\hline
\end{tabular}




\begin{tabular}{|c|c|c|c|c|c|c|c|}
\hline 17 & I $\sigma \pi \alpha v i ́ \alpha$ & $>595$ & 67 & $<11,3 \%$ & $>3$ & 0 & \\
\hline 18 & 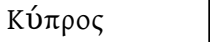 & $>575$ & 382 & $<66,4 \%$ & $>2$ & 0 & \\
\hline 19 & 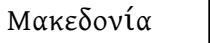 & $>816$ & 296 & $<36,3 \%$ & $>4$ & 0 & \\
\hline 20 & 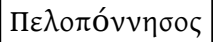 & $>748$ & 292 & $<39 \%$ & $>2$ & 0 & \\
\hline 21 & 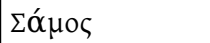 & $>308$ & 138 & $<44,8 \%$ & $>0$ & 0 & \\
\hline 22 & 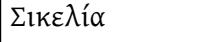 & > 1388 & 389 & $<28 \%$ & $>5$ & 0 & \\
\hline & moyenne & > 1016 & 332 & non signif. & $>4,66$ & 0,56 & non signif. \\
\hline
\end{tabular}

Tab. II. Tableau des notices incomplètes.

42 Sur cette base, il a été possible d'étudier de manière plus précise, pour chaque notice, le rapport entre le nombre de caractères chez Stéphane de Byzance et dans l'Épitomé, qui correspond au taux de réduction du texte (fig. 1). On distingue deux groupes très homogènes (coefficient de corrélation supérieur à 0,90 ) : un premier groupe ${ }^{40}$ avec un facteur d'abréviation moyen proche de $70 \%$, et un second ${ }^{41}$ avec un facteur moyen voisin de $87,5 \%$.

Dans les graphiques qui suivent, nous avons représenté de façon particulière les notices du second groupe, connues par Constantin Porphyrogénète. Après le numéro de la notice est placée une flèche signalant que le positionnement peut être amené à se déplacer vers la droite, en fonction du nombre de caractères réel (mais inconnu) de la notice d'origine. 
Compte tenu de cette incertitude, nous avons surtout fondé notre interprétation sur les notices du premier groupe.

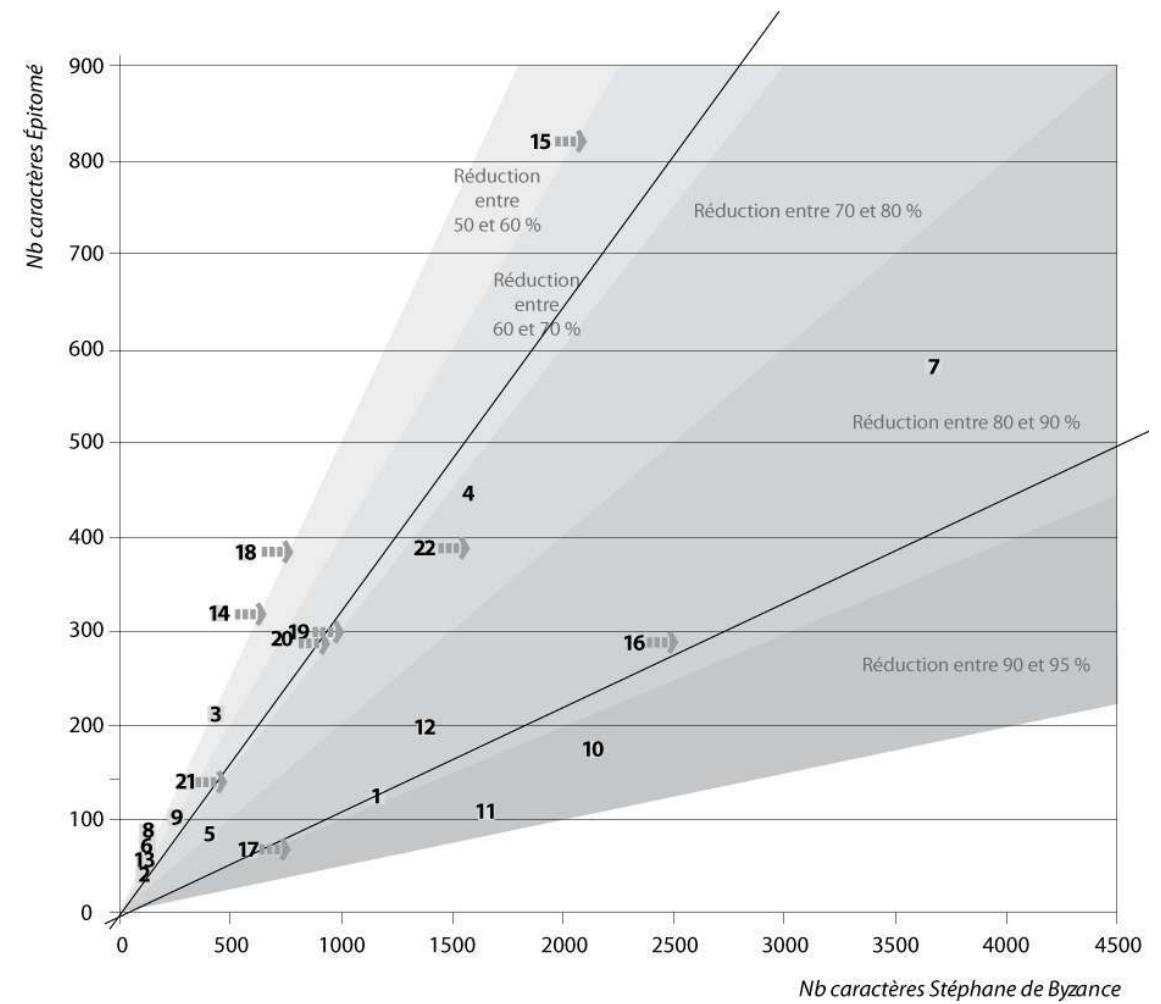

Fig. 1. Rapport entre le nombre de caractères par notice chez Stéphane de Byzance et l'Épitomé et droites de corrélation des deux groupes.

Deux notices apparaissent isolées : celle de $\Delta \tilde{\omega} \rho \circ{ }^{\circ}\left(n^{\circ} 11\right)$ qui contient une citation de Claudius Iolaus ${ }^{42}$ longue de 10 lignes; si l'on en fait abstraction, la notice peut être rattachée au second groupe. Quant à la notice de $\Delta \omega \dot{p}$ iov, elle est lacunaire dans le manuscrit; le nombre de caractères tel qu'il peut être restitué est plutôt hypothétique. L'échelle de la fig. 2, de type logarithmique, accentue l'importance de l'abréviation. Elle met bien en évidence la plus grande réduction des toponymes les plus connus. 


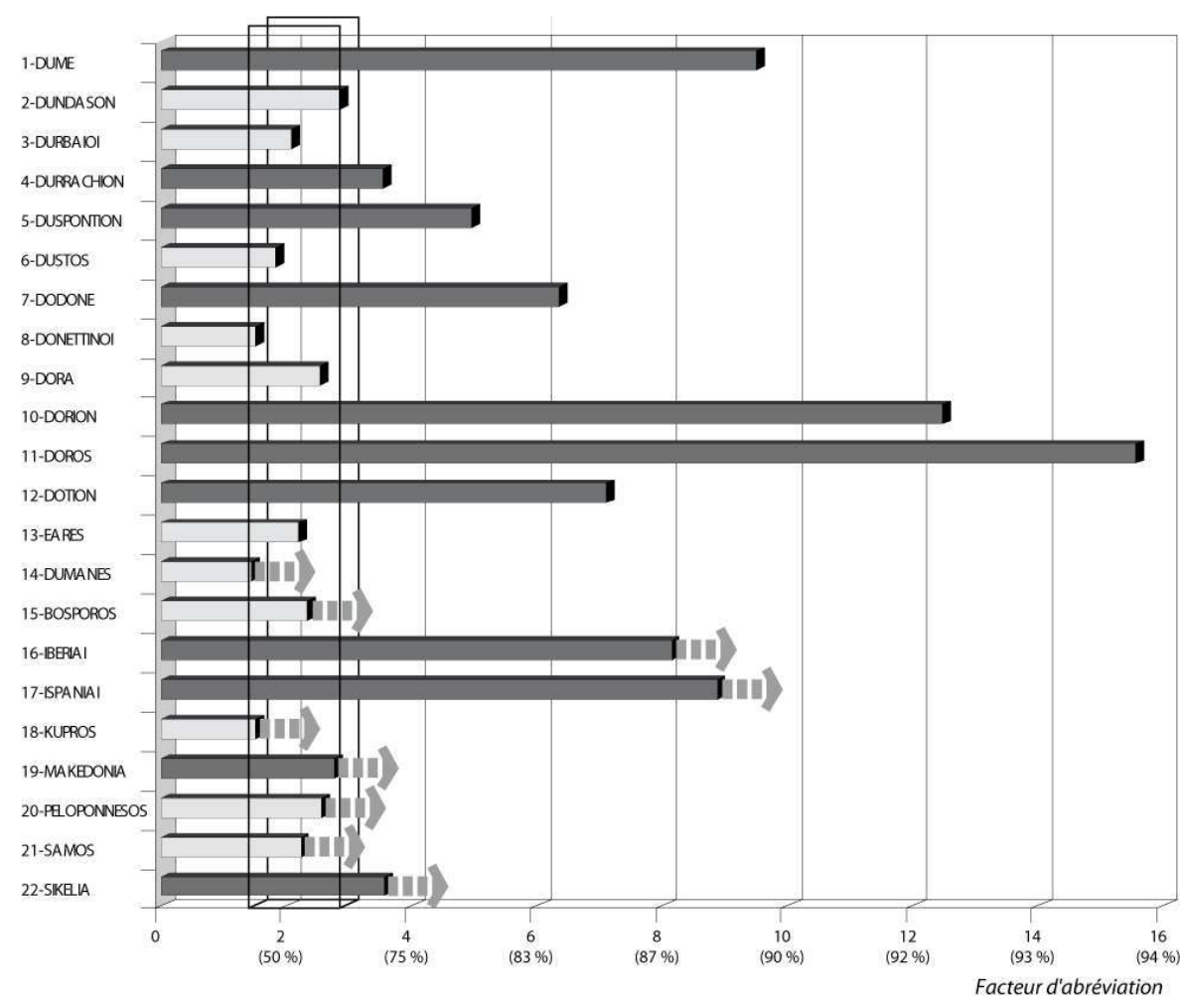

Fig. 2. Facteur d'abréviation des notices.

Comme nous allons le voir plus loin, la cause de cette différenciation des notices en deux groupes apparaît en examinant le graphique de la fig. 4 : fort logiquement, ce sont les notices qui ont le plus de citations chez Stéphane de Byzance qui sont abrégées d'un facteur compris entre 6 et 8.

On remarque également, et là encore il n'y a rien d'anormal, que les petites notices (moins de 10 lignes), sont proportionnellement moins réduites dans l'Épitomé.

Au final, les notices de l'Épitomé n'ont que quelques lignes; sur les 13 notices étudiées, la moyenne est de 175 caractères (239 pour les 22 notices conservées), à comparer aux 1020 caractères moyens (505 pour les 22 notices) chez Stéphane de Byzance. C'est effectivement la longueur moyenne que l'on repère lorsque l'on consulte l'édition de Meineke.

Le facteur de réduction moyen général semble compris entre 5 et $6^{43}$. La version éditée par A. Meineke contient 561345 caractères pour 713 pages dont environ un tiers d'apparat critique (soit un total d'environ 475 pages). Si l'on extrapole le facteur de réduction d'environ 5,6 à l'ensemble de l'ouvrage, il faut imaginer que la version initiale représentait l'équivalent d'environ 2660 pages imprimées pour le seul texte de Stéphane de Byzance, ce qui en faisait une œuvre véritablement hors norme. On comprend mieux ainsi qu'un abrégé ait été rédigé ; un manuscrit aussi imposant n'a pas dû être recopié souvent. Seule Constantinople devait en posséder un (des?) exemplaire(s), ce qui explique l'usage qu'a pu en faire Constantin VII Porphyrogénète et le faible nombre de citations de l'œuvre d'origine (aucune en dehors du cercle des intellectuels constantinopolitains). Nous reviendrons plus en détail sur tout ceci (§ 3.1). 


\subsubsection{La disparition des citations}

Compte tenu de la façon dont l'ouvrage a été organisé, le nombre de citations et le nombre de caractères sont logiquement liés. Afin de préciser cette interaction nous avons établi deux graphiques étudiant chez Stéphane de Byzance et l'Épitomé le rapport entre le nombre de caractères de la notice et le nombre de citations qu'elle contient (quelle que soit leur longueur).

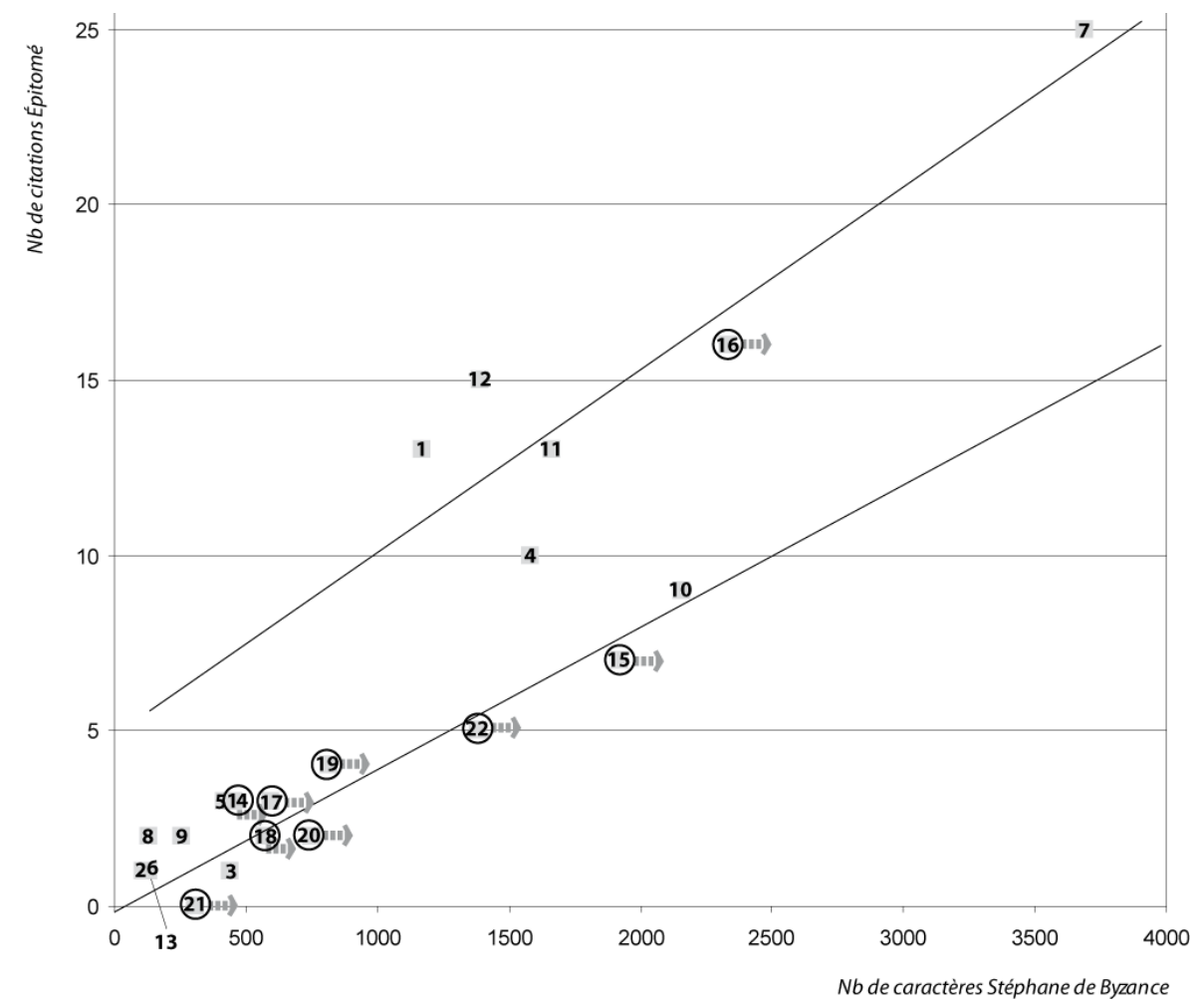

Fig. 3. Rapport entre le nombre de caractères et le nombre de citations chez Stéphane de Byzance et droites de corrélation des deux groupes.

Chez Stéphane de Byzance (fig. 3), le nuage de points est regroupé autour de deux droites de corrélation. La première (coefficient de corrélation: 0,94) rassemble les notices contenant le plus de citations ${ }^{44}$. La deuxième droite regroupe les notices relativement brèves (moins de 20 lignes) et 3 notices un peu longues (coefficient de corrélation 0,91$)^{45}$. Ces courtes notices, qui forment un groupe relativement homogène, n'ont que très peu de citations. Au-delà de 20 lignes, le nombre de citations est beaucoup plus important et suit un accroissement linéaire.

51 Ces droites de corrélation démontrent le lien intrinsèque entre les notices et les citations ; ces dernières en constituent une part significative.

Pour l'Épitomé en revanche, il n'existe que peu de cohérence d'ensemble : le coefficient de corrélation global est seulement de 0,50. On remarquera que l'Épitomé ne mentionne guère plus de deux citations dans les articles étudiés ${ }^{46}$. On a perdu ici le lien fort de l'œuvre originale, qui unissait le nombre de citations à la longueur des notices. C'est donc avant tout sur ces citations qu'a porté la réduction opérée sur l'œuvre de Stéphane de Byzance. Or, ce sont elles qui permettent une étude historique des toponymes. 

citations dans l'œuvre de Stéphane de Byzance et leur nombre dans l'Épitomé. Le pourcentage de citations perdues est considérable. On peut admettre que dans l'ensemble, plus des $3 / 4$ des citations de Stéphane de Byzance ont disparu avec l'abréviation ${ }^{47}$.

On s'aperçoit par ailleurs qu'il n'existe pas de rapport entre le nombre de citations chez Stéphane de Byzance et celui qui subsiste dans les notices compilées. Dans une notice comme celle de $\Delta \omega \delta \omega ́ v \eta$ qui contient 21 citations à l'origine, le compilateur n'en laisse que deux.

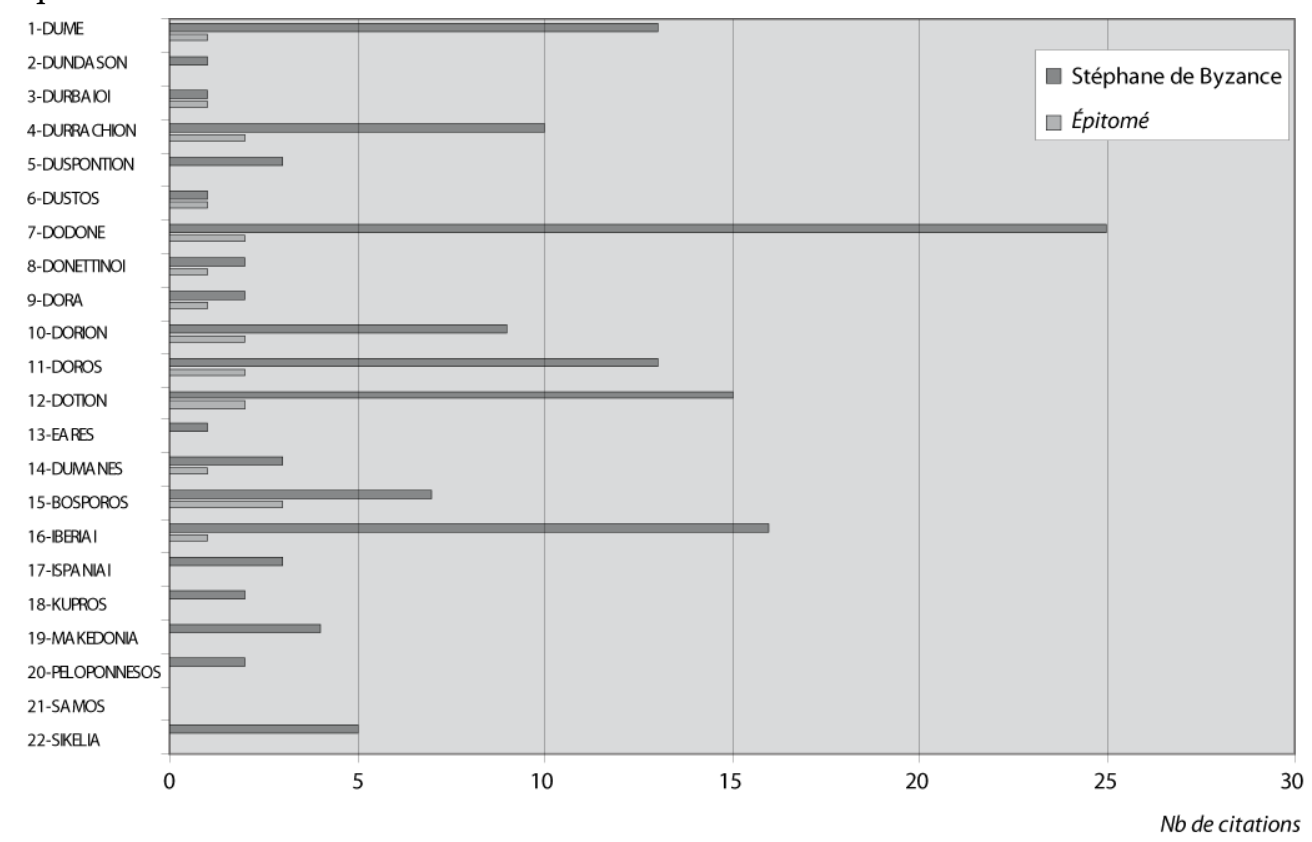

Fig. 4. Comparaison du nombre de citations chez Stéphane de Byzance et l'Épitomé.

\subsection{Vers une restitution de l'ampleur de l'œuvre d'origine}

Pour mieux approcher la taille de l'œuvre d'origine, il est nécessaire de connaître avec plus de précision la réduction opérée. Nous allons l'analyser de deux manières différentes. D'abord en essayant de déterminer si l'abréviation est identique d'une lettre à l'autre. Ensuite en affinant cette recherche dans le cadre du découpage initial de l'œuvre en livres.

Lorsque l'on consulte la totalité de l'ouvrage, on s'aperçoit que certaines lettres ont des notices plus réduites que d'autres, ce qu'avaient déjà notés Westermann puis Honigmann ${ }^{48}$. La moyenne générale de toutes les lettres s'établit à 151 caractères par notice, ce qui correspond à une notice fictive qui nous servira de témoin pour mieux apprécier le taux de réduction de telle ou telle partie de l'ouvrage.

\subsubsection{L'ouvrage et ses divisions}

Des indications de changement de livres sont conservées partiellement dans quatre manuscrits des $E$ thnika ${ }^{49}$. Jusqu'à présent, la plupart des érudits ont considéré que le nombre de livres supposé (60) posait problème ${ }^{50}$. E. Honigmann lui-même donne un tableau récapitulatif - dont on sent bien qu'il lui parait peu logique -, en prenant pour 
base le nombre de notices par livre ${ }^{51}$. Or, leur nombre est très inégal suivant les lettres. De plus, la réduction plus importante de certaines lettres permet d'expliquer les difficultés qu'a rencontrées E. Honigmann dans la restitution des 24 derniers livres dont on perdu l' incipit.

Dans le manuscrit que nous avons conservé (Coislinianus 228, c'est-à-dire le fragment de l'œuvre intégrale), nous avons l'indication d'un changement de livre. Si rien ne signale la fin du livre XIII, apparait, en tête du livre suivant (XIV), le titre complet de l'ouvrage, puis le numéro du livre (sous la forme très simple de " $\beta \imath \beta \lambda$ íov $\left.{ }_{1} \delta^{\prime} »\right)$ suivi de l'index (pinax). Il semble que ce soit ainsi que chaque livre débutait.

Dans les différents manuscrits conservés de notre épitomé, des formes différentes apparaissent. Elles mentionnent soit le début soit la fin d'un livre ou bien d'une lettre.

Il faut d'abord signaler quelques erreurs dans le changement de livre. La première, la plus facile à mettre en évidence, correspond au passage du livre XIV à XV. Dans les manuscrits, l'indication est placée après le lemme "E $\lambda$ upo $(\varepsilon 75)$. Or, nous savons par l'index contenu dans le fragment original du livre XIV que ce livre ce terminait avec la notice suivante "E $\lambda \omega \rho \circ \varsigma$ ( $\varepsilon 76$ ), ce qui est plus logique d'un point de vue alphabétique. Il en est de même du début du livre XXI (Iкóৎ [1 47]au lieu de 'I $\lambda \alpha \rho \alpha u \gamma \tilde{\alpha} \tau \alpha 1$ [1 48]), et du

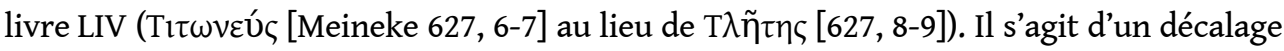
somme toute très modeste, ce qui semble indiquer que déjà dans le manuscrit antérieur, les indications de changement de livre devaient se trouver en marge et qu'ils ont été mal lus.

61 Le numéro de livres est incertain pour la fin de la lettre $\Sigma$ et la lettre T. Si le livre LI ( $\left.v \alpha^{\prime}\right)$ est exact, celui qui commence par Tó $\beta \alpha_{l}$ (Meineke 597, 6) doit correspondre au LII $\left(v \beta^{\prime}\right.$ au lieu de $\left.v \delta^{\prime}\right)$. Nous verrons plus loin que c'est vraisemblablement ce numéro de livre qui est erroné. livres et des livres XXXI à XXXVI, avec les entrées alphabétiques concernées et le nombre de caractères dans l'édition de Meineke. On l'a vu, ces livres ne posent pas de problème de délimitation : le début de chacun est clairement énoncé dans les manuscrits ${ }^{52}$. Ce premier échantillon permet d'inclure dans notre réflexion le livre XIII qui contient les notices dont nous avons conservé l'original. Dans une colonne spécifique, nous avons signalé le nombre de caractères moyen par notice et le rapport (sous forme de \%) entre celui-ci et notre longueur « témoin » (151 caractères). 


\begin{tabular}{|c|c|c|c|c|c|c|}
\hline $\begin{array}{l}\mathrm{N}^{\circ} \mathrm{du} \\
\text { livre }\end{array}$ & $\begin{array}{c}\text { Début } \\
\text { du } \\
\text { livre }\end{array}$ & $\begin{array}{c}\text { Index } \\
\text { géographique }\end{array}$ & $\begin{array}{c}\text { nb de } \\
\text { caractères } \\
\text { dans l' } \\
\text { Épitomé }\end{array}$ & $\begin{array}{l}\mathrm{Nb} \text { de } \\
\text { notices }\end{array}$ & $\begin{array}{c}\text { Nb moyen } \\
\text { de caractère } \\
\text { par notice }\end{array}$ & $\begin{array}{c}\text { \% de longueur } \\
\text { des notices par } \\
\text { rapport à la } \\
\text { longueur } \\
\text { moyenne }\end{array}$ \\
\hline $\mathbf{I}$ & A & $\begin{array}{l}\text { 'A } \beta \alpha \imath \\
A \delta v \rho \mu \alpha x i ́ \delta \alpha 1\end{array}$ & 23393 & 68 & 344 & $+127,8 \%$ \\
\hline II & $\mathrm{A} \varepsilon$ & 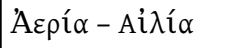 & 15166 & 59 & 257 & $+70,2 \%$ \\
\hline III & Aı $\mu$ & Aipoví $\alpha-A \lambda \varepsilon^{\alpha} \alpha$ & 17226 & 69 & 250 & $+65,3 \%$ \\
\hline IV & $\mathrm{A} \lambda \varepsilon \xi$ & 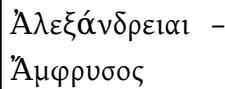 & 17554 & 99 & 177 & $+17,4 \%$ \\
\hline v & $A v$ & 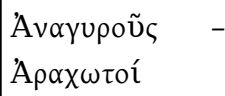 & 15967 & 85 & 188 & $+24,4 \%$ \\
\hline VI & $A \rho \beta$ & $\begin{array}{l}\text { A } \rho \beta \alpha ́ k \eta \\
\text { 'А } \rho \omega \mu \alpha\end{array}$ & 14067 & 84 & 167 & $+10,9 \%$ \\
\hline VII & $A \sigma$ & 'A $\sigma \alpha i ́$ - 'А $\omega \rho о \varsigma$ & 19696 & 113 & 174 & $+15,4 \%$ \\
\hline VIII & B & 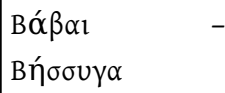 & 11599 & 90 & 129 & $-14,7 \%$ \\
\hline IX & $\mathrm{Bl}$ & 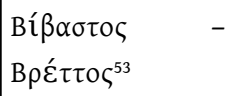 & 14781 & 80 & 197 & $+22,4 \%$ \\
\hline $\mathrm{x}$ & B $\rho 1$ & 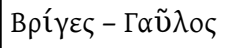 & 11856 & 71 & 167 & $+10,6 \%$ \\
\hline XI & $\Gamma \varepsilon$ & 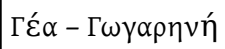 & 12126 & 81 & 150 & $-0,9 \%$ \\
\hline XII & $\Delta$ & $\Delta \alpha ́ \alpha \alpha 1-\Delta i ́ k \tau \eta$ & 12247 & 82 & 149 & $-1,1 \%$ \\
\hline XIII & $\Delta \mathrm{lv}$ & $\begin{array}{l}\Delta \mathfrak{\imath} \delta \delta \rho \tilde{u} \mu \eta \\
\Delta \omega \dot{\omega} \iota v^{54}\end{array}$ & 9500 & 69 & 297 & $-8,8 \%$ \\
\hline XIV & E & $\begin{array}{l}\text { "E } \alpha \rho \varepsilon \varsigma \\
" E \lambda \omega \rho \circ \varsigma^{55}\end{array}$ & 7973 & 74 & 109 & $-28,6 \%$ \\
\hline $\mathbf{X V}$ & $E \mu$ & 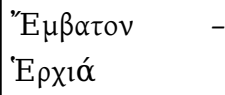 & 9013 & 62 & 145 & $-3,7 \%$ \\
\hline XVI & E $\sigma$ & 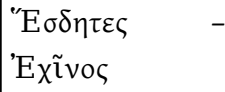 & 7578 & 48 & 158 & $+4,6 \%$ \\
\hline XVII & Z & $\begin{array}{l}\text { Zó } \beta 1 \delta \alpha \\
Z \omega \sigma \tau \eta ́ p\end{array}$ & 4581 & 34 & 135 & $-10,8 \%$ \\
\hline
\end{tabular}




\begin{tabular}{|c|c|c|c|c|c|c|}
\hline XVIII & $\mathrm{H}$ & 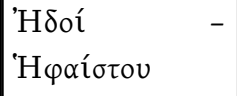 & 5089 & 30 & 170 & $+12,3 \%$ \\
\hline XIX & $\Theta$ & $\begin{array}{l}\Theta \alpha \lambda \alpha^{\prime} \mu \alpha 1 \\
\Theta \omega ́ \rho \alpha \xi\end{array}$ & 11332 & 82 & 138 & $-8,5 \%$ \\
\hline $\mathbf{x X}$ & I & ”I $\alpha \beta ı \varsigma$ - 'Ікóvıv & 7140 & 45 & 159 & $+5,1 \%$ \\
\hline XXI & $\mathrm{I} \lambda$ & $\begin{array}{l}\text { 'I } \lambda \alpha \rho \alpha v \gamma \tilde{\alpha} \tau \alpha 1 \quad- \\
\text { 'I } \omega \tau \alpha \dot{\alpha} \pi \alpha \tau \alpha\end{array}$ & 11010 & 85 & 130 & $-14,2 \%$ \\
\hline XXXI & $\mathrm{M} \varepsilon$ & 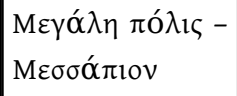 & 7558 & 58 & 130 & $-13,7 \%$ \\
\hline XXXII & 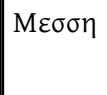 & 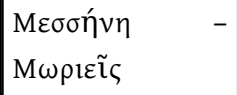 & 14167 & 109 & 130 & $-13,9 \%$ \\
\hline XXXIII & $\mathrm{N}$ & 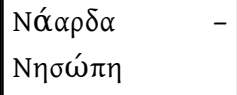 & 6162 & 49 & 126 & $-16,7 \%$ \\
\hline XXXIV & $\mathrm{Ni}$ & 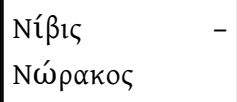 & 5203 & 40 & 130 & $-13,9 \%$ \\
\hline XXXV & $\Xi$ & $\Xi \alpha ́ v \theta o l-\Xi u ́ \sigma \tau 1 \zeta$ & 1382 & 16 & 86 & $-42,8 \%$ \\
\hline XXXVI & 0 & 'O $\alpha-\mathrm{O} \rho \varepsilon \sigma \tau i ́ \alpha$ & 10718 & 88 & 122 & $-19,3 \%$ \\
\hline
\end{tabular}

Tab. III. Tableau de comptage des livres I à XXI et XXXI à XXXVI.

Nous constatons d'abord une différence de réduction du texte original très nette suivant les livres. Nous ne considérerons pas comme très significative toute réduction comprise entre $-15 \%$ et $+15 \%$ par rapport à notre moyenne-pivot, compte tenu du manque de points de comparaison avec l'œuvre d'origine.

Les trois premiers livres sont relativement peu réduits (mais cela représente tout de même une perte allant des trois au quatre-cinquièmes du texte d'origine); ce type de réduction "légère " n'a concerné à peu près que les citations. Suivent ensuite quatre autres livres pour le reste de la lettre $\alpha$ à peine moins réduits que la moyenne générale. Pour les lettres $\beta$ à $\delta$, les livres sont à peu près équivalents en termes de réduction, même si le livre IX semble un peu moins réduit et le livre VIII plus réduit que la moyenne. Le livre XXI et les livres XXXI-XXXIV et XXXVI ont un taux de conservation de texte relativement semblable.

Ce tableau montre l'intérêt de raisonner en fonction des livres d'origine. Le découpage que l'on a conservé par exemple pour la lettre $\alpha$ implique qu'une même lettre pouvait être répartie entre plusieurs livres, en fonction de la place disponible. Le nombre de livres de l'œuvre d'origine (très vraisemblablement 60 si l'on tient compte des livres LI et suivants) ne nous semble pas anormal. E. HonigmanN (1929: col. 2378-2379) pensait le contraire; il a essayé de les retrouver en fonction du nombre de notices de l'Épitomé par livre ce qui nous semble moins pertinent ${ }^{56}$. Quoiqu'il en soit, le découpage enlivres est autant que possible logique (lié à l'ordre alphabétique) mais n'était sans doute pas destiné 
à équilibrer entre eux les différents livres ${ }^{57}$. Pour ne prendre que deux exemples, le livre XVII pour la lettre $\mathrm{H}$ ou XXXV pour la lettre $\Xi$ semblent avoir été beaucoup plus petits que les autres.

Plusieurs lacunes dans l'ordre alphabétique des notices de l'Épitomé ont été identifiées et

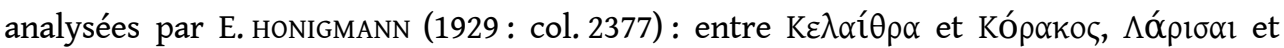

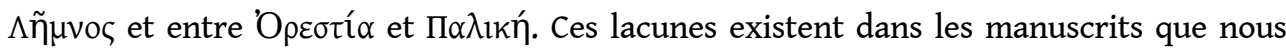
possédons. Ou bien leur archétype avait perdu les feuillets correspondant ou la disparition du texte remonte à un état encore antérieur. En général, les disparitions que l'on observe correspondent plutôt à des pertes de feuillets au début ou à la fin des manuscrits, plus rarement de cahiers entiers au milieu. Il s'agit ici vraisemblablement d'un nombre élevé de feuillets qui correspondent à un ou plusieurs cahiers. Au vu de la façon dont Stéphane de Byzance a découpé les lettres comportant le plus de notices, nous pensons qu'il peut s'agir de livres entiers de Stéphane de Byzance (et/ou de son premier abréviateur puisque celui-ci semble avoir gardé le découpage primitif). Nous allons partir de cette hypothèse de travail ; nous y reviendrons à la fin de notre analyse. En fonction de ces absences, nous avons essayé de répartir les livres en tenant compte des indications des manuscrits ${ }^{58}$. Dans le tableau qui suit (tab. IV), nous avons indiqué en italique les numéros des livres XXII à LX qui restent hypothétiques et entre crochets les lemmes débutant ou finissant un de ces livres restitués. Rappelons que le découpage des livres non signalé de façon spécifique, reste évidemment hypothétique.

\begin{tabular}{|c|c|c|c|c|c|c|}
\hline $\begin{array}{l}N^{\circ} \text { du } \\
\text { livre }\end{array}$ & $\begin{array}{c}\text { Début } \\
\text { du } \\
\text { livre }\end{array}$ & $\begin{array}{c}\text { Index } \\
\text { géographique }\end{array}$ & $\begin{array}{c}\text { nb de } \\
\text { caractères } \\
\text { dans l' } \\
\text { Épitomé }\end{array}$ & $\begin{array}{l}\mathrm{Nb} \text { de } \\
\text { notices }\end{array}$ & 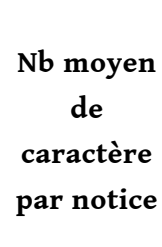 & $\begin{array}{c}\text { \% de longueur } \\
\text { des notices } \\
\text { par rapport à } \\
\text { la longueur } \\
\text { moyenne }\end{array}$ \\
\hline XXII & $\mathrm{K}$ & 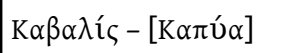 & 9391 & 70 & 134 & $-11,2 \%$ \\
\hline XXIII & $K \alpha \rho$ & 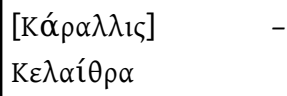 & 13452 & 84 & 160 & $+6,1 \%$ \\
\hline XXIV & $\operatorname{K\varepsilon } \lambda \beta$ & {$[K \varepsilon \lambda \beta \ldots . .]-.[K o \pi$...] } & disparu & & & \\
\hline $\mathrm{XXV}$ & Kop & 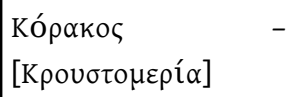 & 11610 & 80 & 145 & $-3,9 \%$ \\
\hline XXVI & K $\rho v$ & 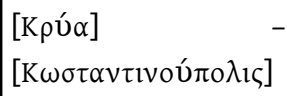 & 13080 & 85 & 154 & $+1,9 \%$ \\
\hline XXVII & $\Lambda$ & {$[\Lambda \tilde{\alpha}]-[\Lambda \alpha ́ \rho \mid \sigma \alpha 1 \ldots]$} & 7153 & 45 & 159 & $+5,3 \%$ \\
\hline XXVIII & $\Lambda \alpha \sigma$ & {$[\Lambda \alpha \sigma \quad \ldots]-[\Lambda \eta \ldots]$} & disparu & & & \\
\hline XXIX & $\Lambda \eta \mu$ & $\begin{array}{l}{[\Lambda \tilde{n} \mu \nu \circ \varsigma]} \\
{\left[\Lambda \omega \tau \circ \varphi \operatorname{\rho o}_{\rho} \circ \varsigma\right]}\end{array}$ & 7672 & 75 & 102 & $-32,3 \%$ \\
\hline $\mathrm{XXX}$ & M & 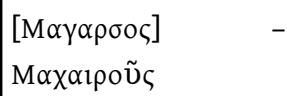 & 10222 & 103 & 99 & $-34,3 \%$ \\
\hline
\end{tabular}




\begin{tabular}{|c|c|c|c|c|c|c|}
\hline XXXI & $\mathrm{M \varepsilon}$ & 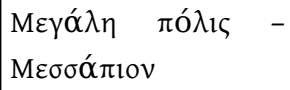 & 7558 & 58 & 130 & $-13,7 \%$ \\
\hline XXXII & 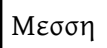 & 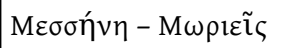 & 14167 & 109 & 130 & $-13,9 \%$ \\
\hline XXXIII & $\mathrm{N}$ & Nó $\alpha \rho \delta \alpha-N \eta \sigma \omega ́ \pi \eta$ & 6162 & 49 & 126 & $-16,7 \%$ \\
\hline XXXIV & $\mathrm{Nl}$ & 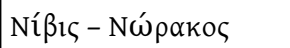 & 5203 & 40 & 130 & $-13,9 \%$ \\
\hline XXXV & $\Xi$ & $\Xi \alpha ́ v \theta o l-\Xi u ́ \sigma \tau 1 \varsigma$ & 1382 & 16 & 86 & $-42,8 \%$ \\
\hline XXXVI & 0 & 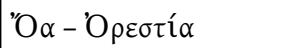 & 10718 & 88 & 122 & $-19,3 \%$ \\
\hline XXXVII & Opl & {$[\mathrm{O} \rho 1 \ldots . .]-.[0 \psi \ldots]$} & disparu & & & \\
\hline XXXVIII & $\Pi$ & 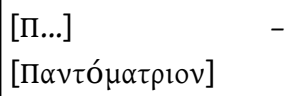 & > 4989 & $>27$ & (185) & $+22,4 \%$ \\
\hline XXXIX & $\Pi \alpha \pi$ & 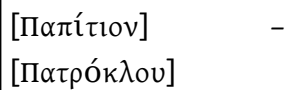 & 8947 & 46 & 195 & $+28,8 \%$ \\
\hline$X L$ & $\Pi \alpha \tau v$ & 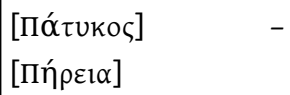 & 7601 & 70 & 109 & $-28,1 \%$ \\
\hline$X L I$ & Пı & 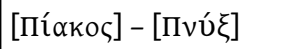 & 4789 & 45 & 106 & $-29,5 \%$ \\
\hline XLII & По & 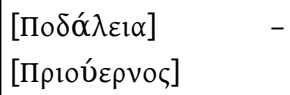 & 3875 & 53 & 73 & $-51,6 \%$ \\
\hline XLIII & Про & 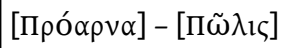 & 4899 & 54 & 91 & $-39,9 \%$ \\
\hline XLIV & $\mathrm{P}$ & 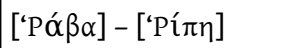 & 2833 & 36 & 79 & $-47,9 \%$ \\
\hline$X L V$ & Po & 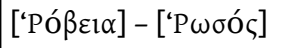 & 1875 & 29 & 65 & $-57,2 \%$ \\
\hline$X L V I$ & $\Sigma$ & {$[\Sigma \alpha \beta \alpha i ́]-\left[\Sigma \alpha^{\prime} \mu \psi \varepsilon ı \rho \alpha\right]$} & 3700 & 48 & 77 & $-49 \%$ \\
\hline$X L V I I$ & $\Sigma \alpha v$ & 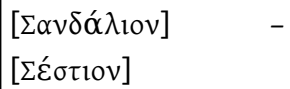 & 5288 & 64 & 83 & $-45,3 \%$ \\
\hline XLVIII & $\Sigma \eta$ & $\begin{array}{l}{[\Sigma \eta \kappa o \alpha v o ́ \varsigma]} \\
{[\Sigma ı \omega v i ́ \alpha]}\end{array}$ & 8073 & 76 & 106 & $-38,3 \%$ \\
\hline XLIX & $\Sigma \kappa$ & 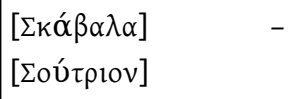 & 7063 & 64 & 110 & $-26,9 \%$ \\
\hline$L$ & $\Sigma \pi$ & $\begin{array}{l}{[\Sigma \pi \alpha ́ \alpha \delta]} \\
\Sigma v \pi \alpha \lambda \eta \tau \tau o ́ \varsigma\end{array}$ & 7745 & 67 & 146 & $-3,3 \%$ \\
\hline LI & $\Sigma u \rho$ & $\begin{array}{l}\Sigma v \rho \alpha ́ \alpha \kappa v \sigma \alpha \imath \\
\Sigma \omega \varphi \eta v \eta ́\end{array}$ & 4165 & 23 & 181 & $+19,9 \%$ \\
\hline
\end{tabular}




\begin{tabular}{|c|c|c|c|c|c|c|}
\hline [LII] & $\mathrm{T}$ & Tó $\beta \alpha 1-T \tilde{\eta} v o \varsigma$ & 21011 & 116 & 181 & $+20 \%$ \\
\hline [LIII] & $\mathrm{T} 1$ & 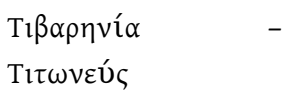 & 3639 & 26 & 140 & $-7,3 \%$ \\
\hline [LIV] & $\mathrm{T} \lambda$ & 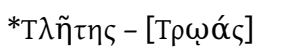 & 9544 & 69 & 138 & $-8,4 \%$ \\
\hline$L V$ & Tu & [Tú $\alpha v \alpha]-\left[Y \omega^{\prime} \pi \eta\right]$ & 11876 & 84 & 141 & $-6,4 \%$ \\
\hline$L V I$ & $\Phi$ & 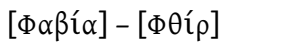 & 8996 & 59 & 152 & $+1 \%$ \\
\hline LVII & $\Phi 1$ & 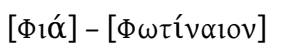 & 10034 & 64 & 157 & $+3,8 \%$ \\
\hline LVIII & $\mathrm{X}$ & 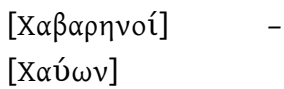 & 10289 & 34 & 303 & $+100,4 \%$ \\
\hline LIX & $\mathrm{X} \varepsilon$ & 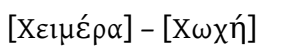 & 8689 & 33 & 263 & $+74,4 \%$ \\
\hline$L X$ & $\Psi$ & {$[\Psi]-\tilde{\Omega} \varphi \theta_{1 \varsigma}$} & 10855 & 46 & 236 & $+56,3 \%$ \\
\hline
\end{tabular}

Tab. IV. Proposition de restitution des livres XXII à LX de Stéphane de Byzance.

72 Nous proposons de voir dans les lacunes repérées dans le texte les livres XXIV, XXVIII et XXXVII. Ces manques ne sont en effet pas compatibles avec un découpage du manuscrit en plusieurs volumes dont les derniers (ou premiers) folios auraient disparu: les lacunes auraient été alors relevées selon un rythme régulier. Ces intervalles entre les lemmes restent compatibles avec les longueurs moyennes des séquences alphabétiques. Jusqu'au livre XXXVI, on a un découpage assez cohérent ; au-delà, il devient plus difficile à établir. Le livre XXXVII, disparu, pourrait avoir compris la fin de la lettre $O$, avec les très nombreux noms d'origine latine débutant par OỦ-. Dès lors, le début de la lettre $\Pi$ se serait trouvé dans le livre suivant dont les premiers folios auraient disparu. La lettre $\Pi$ aurait été découpée en 7 livres ; la lettre $P$ en seulement 2 livres tandis que l'on retrouve 6 livres pour la lettre $\Sigma$. Le découpage interne de cette dernière lettre est assez difficile à retrouver; nous avons essayé de proposer des césures alphabétiques assez simples, qui restent bien sûr hypothétiques. Pour les derniers livres (de LV à LX) nous avons regroupé la fin de la lettre $\mathrm{T}$ avec le $Y$, et le $\Psi$ avec l' $\Omega$.

On l'a dit, les livres sont inégaux, aussi bien par le nombre de caractères que par celui des notices. Au final, nous pensons que le découpage par livre que nous avons établi ne doit pas être très éloigné de celui de l'œuvre d'origine. Il nous servira pour toute la suite de notre réflexion; il conviendra de garder à l'esprit la limite de nos certitudes concernant cette répartition.

\subsubsection{Une abréviation multiple ?}

Lorsque l'on regarde dans le détail les différents livres, on voit clairement que certains d'entre eux ont fait l'objet d'une réduction différentielle (fig. 5), que met en évidence le pourcentage de la longueur des notices par rapport à la longueur moyenne (établie, rappelons-le à 151 caractères). 


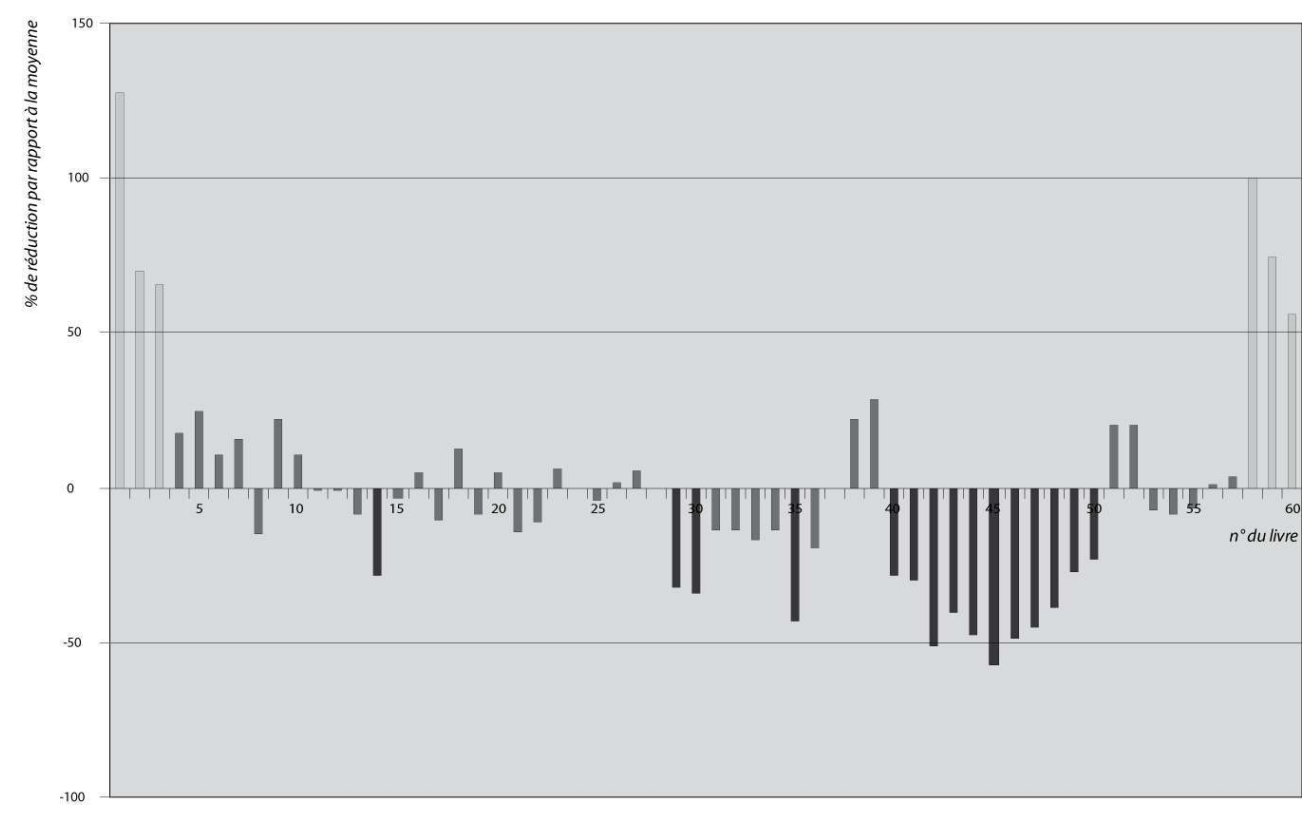

Fig. 5. Pourcentage de réduction des 60 livres de Stéphane de Byzance par rapport à la moyenne.

Les trois premiers et les trois derniers livres sont les moins réduits. La différence est ainsi particulièrement marquante entre le livre LVII (avec une réduction quasi égale à notre moyenne théorique) et le livre LVIII, deux fois moins réduit. Ces deux ensembles de trois livres pourraient indiquer une répartition d'une première phase d'abréviation (que l'on attribuera à un pseudo-Hermolaos, ou H1) regroupant de préférence 3 livres en 1, ce qui nous donne un total de 20 «livres » non attestés, qui est cohérent avec le facteur de réduction moyen de la totalité de l'Épitomé.

Cette répartition par groupe de trois livres est intéressante pour expliquer la disparition de certains livres. Le livre XXIV apparait à la fin d'un groupe de trois ; le livre XXVIII au début d'un tel groupe, de même que le livre XXXVII. Si l'on imagine un regroupement par trois des livres de Stéphane de Byzance après la réduction opérée par le pseudoHermolaos, nous obtenons le schéma suivant (fig. 6), où les livres absents sont effectivement au début ou à la fin des regroupements peut-être effectués par le pseudoHermolaos (noté H1). 


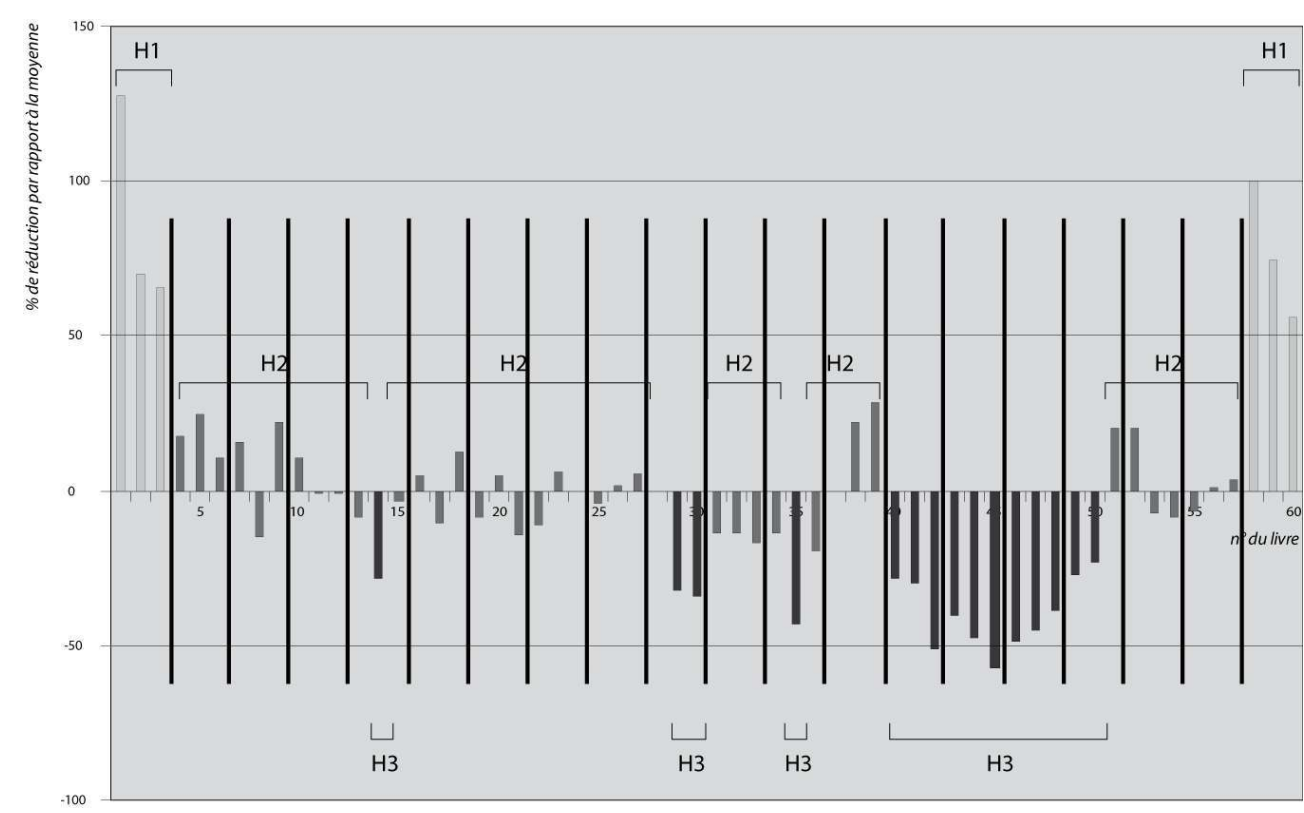

Fig. 6. Proposition de découpage de l'abrégé du pseudo-Hermolaos et les réductions successives.

77 Il nous faut analyser maintenant le pourcentage de réduction que l'on observe pour l'ensemble des autres livres. Si l'on excepte les 3 premiers et les 3 derniers livres, on observe deux séries distinctes. La première a un taux de réduction qui tourne autour de la moyenne ; il s'agit probablement d'une seconde étape dans l'abréviation, réalisée par un inconnu (noté $\mathrm{H} 2$ par la suite).

Les réductions les plus importantes semblent provenir d'un travail de troisième main (noté H3) sur les livres déjà réduits par le second abréviateur. Dans nos manuscrits, ce sont les livres XXVIII-XXX, XXXV, et XL-L qui ont fait l'objet d'une réduction supplémentaire ${ }^{59}$. On notera également, et cela renforce notre hypothèse, que le début de la lettre X, dans l'un des manuscrits (Rehdigeranus 47), présente une forme réduite des 8 premières notices ${ }^{60}$. Nous serions ici en présence à la fois d'un texte du pseudoHermolaos (H1) et de son résumé (H3).

\subsection{La restitution des différentes phases d'abréviation}

Il faut donc imaginer que notre manuscrit archétype était en fait composé à partir de trois manuscrits différents. Le premier est probablement l'abréviation relativement détaillée du pseudo-Hermolaos. Par rapport au découpage de l'œuvre initiale, il ne reste de cet abrégé que les 3 premiers et les 3 derniers livres ; la conservation du texte d'origine dans ce premier épitomé est comprise entre 30 et $40 \%$. Ceci pourrait expliquer le regroupement des livres de Stéphane de Byzance par groupe de trois: la réduction moyenne est à peu près égale à un tiers du texte d'origine. Par ailleurs ce manuscrit restait encore imposant et devait probablement être découpé en plusieurs volumes. C'est sans doute la raison de la perte de certaines parties du texte.

80 La seconde réduction (H2) a réduit encore le texte; il ne reste plus alors qu'entre 15 et $25 \%$ du texte d'origine. Enfin, le dernier résumé (H3) entraine une conservation de seulement 9 à $13 \%$ environ de l'œuvre primitive.

81 La composition de l'ensemble du manuscrit archétype de tous nos manuscrits est donc complexe. Elle reprend des éléments disparates, en essayant de garder le maximum 
d'information. Les parties conservées de l'abrégé du pseudo-Hermolaos (livres I-III et LVIII-LX) sont intégrées en priorité. Dans la partie manquante (livres IV à LVII), le compilateur a privilégié le texte du second abréviateur, mais qui présentait alors des lacunes importantes: sans doute les livres XXIV, XXVIII et XXXVII avaient-ils déjà disparu. Ainsi peut s'expliquer la perte de certaines parties de l'ouvrage du pseudoHermolaos, qu'avait pourtant sous les yeux Eustathe de Thessalonique ${ }^{61}$ : la disparition de feuillets permet d'expliquer les lacunes de la lettre $K$ et $\Lambda$. On note surtout l'absence des livres XXIX, XXX et XL à XLVIII, peut-être XIV et XXXV. Il utilise alors pour remplacer autant que possible ces lacunes une troisième version, très réduite.

Cette composition multiple permet aussi d'expliquer une des variantes signalée plus haut : pour le début du livre LVIII, nous avons conservé une version qui correspond au dernier résumé, à côté des notices très bien conservées. Il est possible que le copiste n'ait pas fait attention qu'il possédait une version encore complète du pseudo-Hermolaos pour ces notices et qu'il ait commencé à copier le résumé. Il aurait ensuite intégré les trois derniers livres du pseudo-Hermolaos. Ou bien on est en présence du verso d'un folio du second abréviateur auquel on a rajouté les derniers cahiers subsistant du pseudoHermolaos. Certains de nos manuscrits, dérivant de notre archétype, auraient alors gardé ou supprimé cette liste qui ne présentait pas d'intérêt.

Ces observations impliquent que quatre versions différentes de l'ensemble de l'œuvre aient existé : celle d'origine dont ne subsiste plus que les feuilles de S. Celle du PseudoHermolaos, qui correspondrait à l'épitomé d'Eustathe de Thessalonique. Un second abrégé aurait été rédigé ensuite (après 1204 ?), suivi lui-même d'une dernière version résumée certainement tardive. Enfin, l'ensemble des «morceaux » subsistant auraient été réunis à un moment donné pour aboutir à l'archétype de nos manuscrits. Nous reviendrons plus en détail sur l'histoire du texte dans le chapitre suivant.

Au vu de tout ce qui précède, il est possible de résumer dans le tableau ci-dessous (tab. V) les informations concernant l'œuvre de Stéphane de Byzance et ses phases successives d'abréviation. Les deux dernières colonnes sont purement hypothétiques : elles donnent une idée du nombre de caractères pour chaque livre chez Stéphane de Byzance $^{62}$ et la taille que cela pouvait représenter en termes de folios dans un manuscrit tardif comme le Perusinus $67^{63}$.

La question qui se pose concerne la taille des livres d'origine. Faut-il imaginer qu'ils avaient à peu près tous la même taille et que le découpage à l'intérieur d'une même lettre est fonction d'un nombre de folios donné ? Ou bien était-il possible que les livres aient eu des tailles totalement différentes? Parmi ceux qui présentent très peu de folios (à 1500 caractères) dans notre hypothèse de restitution, on trouve les livres XXXV (lettre $\Xi$, 6 folios), XLV (début de la lettre P, 11 folios) et XLVI (fin de la lettre P, 8 folios). Pour ce

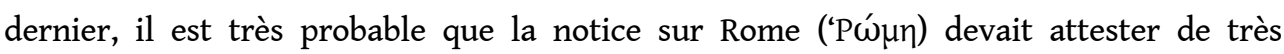
nombreuses formes et que le taux de réduction est de fait beaucoup plus élevé. C'est peutêtre également le cas pour les deux autres livres mentionnés. 


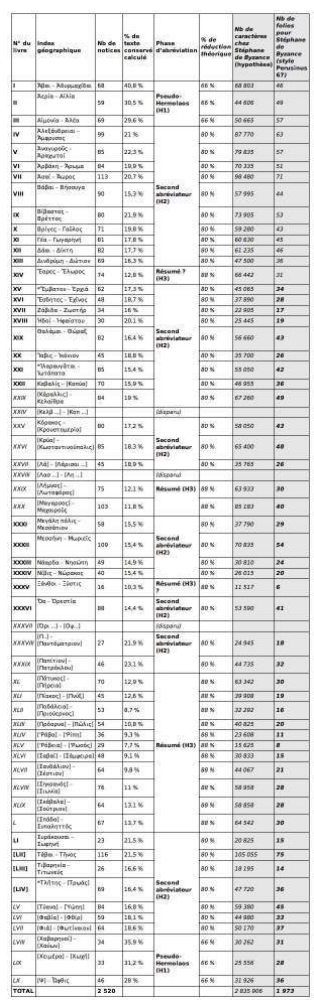

Tab. V. Abréviation et restitution des livres.

Si l'on en revient à la taille de l'ouvrage, on peut donner un ordre d'idée de son ampleur avec le tableau que nous avons dressé. Le manuscrit en miniatures comportait environ 2000 pages soit 1000 folios, ce qui donnerait un manuscrit en onciales du double ${ }^{64}$. Il est tout à fait possible que l'œuvre d'origine de Stéphane de Byzance ait été divisée en 6 manuscrits (de 10 livres chacuns) et sa translittération en 3 (de 20 livres chacun), d'environ 350 folios. Le premier abrégé, en divisant par trois la taille permet de ramener à un seul manuscrit l'ensemble. Il n'est toutefois pas impossible que l'ensemble ait continué à être divisé en trois. Nous reviendrons plus loin plus en détail sur la question de la taille des volumes.

\section{De l'œuvre de Stéphane de Byzance à son Épitomé}

Les études se sont attachées jusqu'à présent à étudier les différents manuscrits connus de l'Épitomé, issus d'un archétype tardif. A. DILLER (1938) les a recensés et sommairement analysés, préparant l'édition critique de M. BILLERBECK et alii (2006: $8^{*}$ sq.) qui les utilise avec efficacité. Nous n'avons rien à ajouter à leurs conclusions. Il est plus pertinent de revoir, au vu de notre étude, ce que l'on peut restituer de l'histoire du texte avant cet archétype.

La confrontation entre le texte de Stéphane de Byzance et l'analyse des réductions successives de l'œuvre permet en effet de préciser les transformations que celle-ci a subies. L'apport principal concerne peut-être la tradition du texte, que l'on peut retracer depuis le manuscrit d'origine jusqu'aux copies de la Renaissance qui nous ont été conservées, en tenant compte de chaque moment où il a été utilisé à l'époque byzantine. Même si les hypothèses sont parfois étayées par peu de preuves, cette histoire reste vraisemblable. 


\subsection{De Stéphane de Byzance à la Souda} période troublée que représente pour Byzance les $\mathrm{VII}^{\mathrm{e}}$ et $\mathrm{VIII}{ }^{\mathrm{e}}$ siècles. On a signalé cidessus ( $\$ 1.1$ ) la mention la plus ancienne que l’on connaisse de Stéphane de Byzance, qui est l'œuvre de ce grammairien ayant enseigné probablement à la fin du viII ${ }^{\mathrm{e}}$ siècle ou au début du siècle suivant ${ }^{68}$. Stéphane de Byzance sert ici de référence pour une règle sur la construction des génitifs des noms propres se terminant par $-\alpha$ (génitif en $-\alpha \varsigma)^{69}$. Les

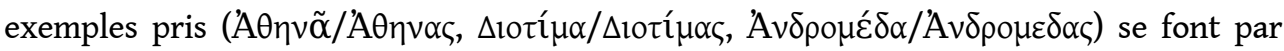

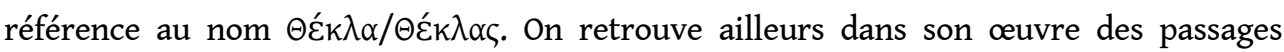
empruntés à Stéphane de Byzance et/ou Hérodien, sans que l'on puisse toujours distinguer l'apport de l'un ou de l'autre du fait de l'importante disparition de l'œuvre de ce dernier. Ainsi, pour certaines villes se terminant par- $-\rho \eta$, Chœroboscos écrit ${ }^{70}$ :

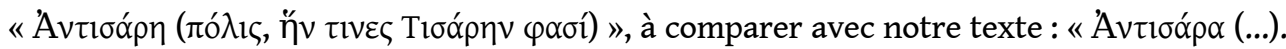

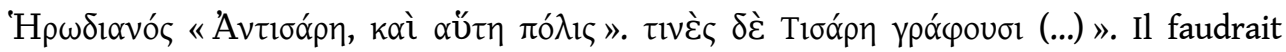
procéder à une vérification systématique dans toute l'œuvre conservée du grammairien mais Georgios Chœroboscos a certainement utilisé assez largement le lexique géographique pour ses œuvres ${ }^{71}$. Il est tout à fait logique qu'un grammairien ait eu un accès à son texte et s'en soit servi comme il le faisait de celui d'Hérodien.

Nous ne trouvons aucune trace de Stéphane de Byzance dans la Bibliothèque de Photios, alors qu'il consacre plusieurs chapitres aux lexiques ${ }^{72}$. Quelques géonymes de son Lexique ${ }^{73}$ présentent une troublante ressemblance avec le texte de Stéphane de Byzance. Or, Photios a utilisé pour son lexique des compilations plus anciennes. C'est donc d'œuvres 
déjà existantes que proviennent ces mentions si proches de celles de Stéphane de Byzance.

Il n'est cependant pas anodin de constater l'utilisation de cet ouvrage par un grammairien (Choeroboscos) autour de 800, alors que Photios quelques décennies plus tard ne mentionne pas les Ethnika et ne l'utilise pas. L'œuvre de Stéphane de Byzance était d'une taille impressionnante et d'un usage vraisemblablement peu commode; c'est un ouvrage de grammairien, pour les grammairiens. Or, Photios ne mentionne aucune œuvre de grammaire dans sa Bibliothèque ou son Lexique. Seuls les lexiques (dont les définitions sont plutôt « courtes " si l'on en juge par sa propre œuvre) trouvent grâce à ses yeux ; l'œuvre de Stéphane de Byzance n'a rien à voir avec ce type d'ouvrages. L'absence d'utilisation des véritables grammariens par Photios est un point qui n'a, à notre connaissance, jamais été mis en avant dans les nombreuses études qui concernent son œuvre et son travail intellectuel. C'est un aspect important dans l'histoire de la transmission des textes antiques et particulièrement dans le cas de Stéphane de Byzance. En effet, il faut se poser la question d'une éventuelle copie du manuscrit dans le nouveau style d'écriture qui se développe à partir des années 810 : la minuscule. Nous l'avons vu supra (\$1.4), le manuscrit d'origine était resté vraisemblablement en l'état et n'a pas été recopié à cette époque ce qui aurait été certainement le cas si Photios l'avait utilisé. Quoiqu'il en soit, c'est certainement dans la bibliothèque impériale que Constantin Porphyrogénète aurait trouvé le manuscrit et l'aurait utilisé.

Le texte original de Stéphane de Byzance est connu de longue date pour avoir servi de source à certaines des œuvres de Constantin VII Porphyrogénète dans le courant du $\mathrm{x}^{\mathrm{e}}$ siècle (Billerbeck et al. 2006, p. 6*). Le De Thematibus a conservé, dans son livre I (daté dans son premier état des années 933/934 par A. Pertusi) et II (daté du milieu du $\mathrm{x}^{\mathrm{e}}$ siècle $\mathrm{e}^{74}$ ) plusieurs extraits d'un texte de Stéphane de Byzance qui semblent relativement complets ${ }^{75}$.

Le De Administrando Imperio (écrit entre 948 et 952$)^{76}$ a réutilisé les deux notices sur l'Espagne ${ }^{77}$; cette œuvre est connue par quatre manuscrits, dont le plus ancien ( Parisinus gr. 2009) date de la fin du XI e siècle (MORAvcsiK, JENKINS, 1967 : 15 sq.). Ce dernier manuscrit est rédigé dans un contexte particulier : comme l'indique la souscription, il a été copié pour un membre de la famille impériale, le César Jean Doukas, frère de l'empereur Constantin X, entre 1059 et 1081. Cet ouvrage a été rédigé par Constantin VII pour l'éducation de son fils, Romain II. Les informations qu'il contient étaient vraisemblablement secrètes ; le livre n'était pas destiné à être édité. Jean Doukas ou l'un de ses conseillers (Michel Psellos?) l'a probablement trouvé dans les archives ou la bibliothèque du palais impérial et l'a fait copier.

Les erreurs du manuscrit sont en grande partie liées à la lecture erronnée du texte primitif de Stéphane de Byzance. Les erreurs sont semblables à celles que l'on trouve dans le Par. Coisl. 228.

Il ne semble pas en revanche que l'ensemble de l'œuvre encyclopédique qu'il a commanditée, les Excerpta Constantiniana (LEMERLE, $1971: 281)^{78}$, ait intégré des fragments des Ethnika; aucun extrait de notre auteur ne nous a été conservé dans les quelques manuscrits rescapés de ce vaste travail ${ }^{79}$. Il semble que seul Constantin ait utilisé ce texte.

101 Dans le courant du $\mathrm{x}^{\mathrm{e}}$ siècle, l'auteur de la chronique anonyme Sur le règne des empereurs, traditionnellement attribuée à Genesios, utilise également Stéphane de Byzance. A. Diller 
a repéré quatre mentions reprenant les notices de Paphlagonia, Amastris, Tarsos et Surakousai $^{80}$. Nous sommes là encore dans l'entourage de Constantin Porphyrogénète ${ }^{81}$.

Nous l'avons dit plus haut (\$1.3) la Souda n’a pas utilisé Stéphane de Byzance, bien qu'elle comporte la mention d'Hermolaos. L'hypothèse la plus vraisemblable concernant la date de l'auteur de cette "abréviation » est qu'il ait été suffisamment éloigné de la date de rédaction de la Souda pour n'être plus qu'un nom trouvé dans une source (l'Onomatologos d'Hesychios de Milet) et non le rédacteur d'un manuscrit que le compilateur avait sous les yeux. La notice sur l'airain de Dodone $(\delta 1445)$ en particulier reprend les termes du seul Photios. De même, on ne retrouve quasiment aucun des géonymes des notices conservées; lorsque les entrées existent, leur texte ne correspond pas à celui de notre auteur. Un exemple particulièrement frappant est celui de la notice Dôros, pourtant développée sur 12 lignes dans la Souda $(\delta 1476)$. Alors que Stéphane de Byzance parle d'une ville de Phénicie, la Souda en fait une ville d'Arabie. Les auteurs cités ne sont pas les mêmes et le discours est différent. Dans la Souda, les toponymes attiques sont souvent issus de l'abrégé d'Harpocration, qui est comme on le verra une source commune avec Stéphane de Byzance ; aucun n'est issu de Stéphane de Byzance, ni de son abréviateur.

Enfin, il faut indiquer les gloses que contiennent plusieurs des manuscrits pour la lettre $\mathrm{N}$, avec de nombreuses mentions rapportées à l'«ethnikographon». Elles sont publiées par Adler en lettres plus petites et reprennent le texte que l'on connait encore dans l'Épitomé, sans pour autant le copier intégralement. Il s'agit d'un rajout tardif attribué de façon certaine à Eustathe de Thessalonique (DILLER, $1938: 335$. ${ }^{82}$.

\subsection{La redécouverte de Stéphane de Byzance au XI ${ }^{\mathrm{e}}$ et dans la $1^{\text {re }}$ moitié du XII ${ }^{\mathrm{e}}$ siècle}

La probable redécouverte du manuscrit, mais plus encore sa copie, dans le courant du XI siècle est fondamentale pour expliquer l'usage que l'on en fait au siècle suivant. L'utilisation la plus importante de l'œuvre de Stéphane de Byzance se place en effet au cours $\mathrm{du} \mathrm{XII}^{\mathrm{e}}$ siècle. C'est probablement au début de cette période qu'intervient la rédaction d'un premier abrégé.

\subsubsection{Le XI ${ }^{\mathrm{e}}$ siècle}

C'est vraisemblablement au $\mathrm{XI}^{\mathrm{e}}$ siècle qu'a été copié le manuscrit [S] dont seuls 8 feuillets nous ont été conservés par le Coislinianus 228. La datation un peu tardive du manuscrit proposée par le seul A. Diller (XII ${ }^{\mathrm{e}}$ siècle) peut s'expliquer par une attribution à une probable seconde moitié du XI ${ }^{\mathrm{e}}$ siècle, sans que l'on puisse donner plus de précision.

Compte tenu de l'œuvre dont il est question (très volumineuse) et des rares mentions que nous en connaissons avant le XII ${ }^{\mathrm{e}}$ siècle, il est très vraisemblable que la copie de Théophile se soit faite directement sur le manuscrit d'origine comme nous l'avons vu supra (§ 1.4). Celui-ci n'aurait donc pas été translittéré au cours des $\mathrm{IX}^{\mathrm{e}}$ et $\mathrm{X}^{\mathrm{e}}$ siècles. Cela semble assez logique pour le $\mathrm{IX}^{\mathrm{e}}$ siècle, si l'on admet l'absence d'attention apportée à cette œuvre par Photios, même si Georgios Choeroboscos l'utilise mais dans un contexte relevant seulement de la science grammaticale. C'est un peu plus étonnant pour l'époque de Constantin Porphyrogénète. Cela explique certainement l'utilisation très ponctuelle du manuscrit à l'époque de cet empereur, et seulement dans son entourage, et son oubli 
rapide. Ainsi dès la fin du siècle, la Souda ne semble déjà plus le connaître ou en tout cas ne l'utilise pas. Le fait qu'il n'ait pas été translittéré a peut-être beaucoup compté de ce point de vue là.

Nous pouvons revenir sur la forme même du manuscrit. Nous avons vu plus haut que le texte de Stéphane de Byzance pouvait comporter selon une approximation toute théorique autour de 2835000 caractères. Le manuscrit de Théophile est composé à raison de 26 lignes par page. Un calcul d'après les folios 120r à 121v (soit 4 pages) permet de proposer un total de 4051 caractères, soit environ 1012 caractères par page et une moyenne de 39 par ligne. Le manuscrit total translittéré pouvait donc comporter autour de 2800 pages $^{83}$. A cela, il faut rajouter, pour chaque livre, quasiment un folio complet pour identifier la fin d'un livre et proposer le pinax du livre suivant, soit 120 pages de plus. Nous arrivons donc à un total (là encore hypothétique, nous le soulignons, mais assez vraisemblable) de 2920 pages soit 1460 folios. Un tel manuscrit était obligatoirement découpé en plusieurs volumes, soit par groupe de six livres (10 manuscrits de 146 folios) soit plus vraisemblablement par groupe de dix livres (6 manuscrits de 243 folios) ${ }^{84}$.

Il faut probablement rectifier la grosseur de chaque volume par le volume d'information que donnait Stéphane de Byzance. Comme on le voit dans le premier abrégé (cf. infra $\S$ 2.3.4), les trois premiers livres étaient deux fois plus importants que les trois derniers. On s'en aperçoit encore à la lecture de l'Épitomé: Stéphane de Byzance donne de nombreuses informations grammaticales au début, qu'il n'a pas forcément reporté dans l'ensemble de son œuvre. Les hypothétiques six volumes étaient donc probablement de taille différente.

109 La translittération d'un tel manuscrit a dû être une opération particulièrement longue, probablement confiée à une équipe de plusieurs copistes, chacun pouvant prendre en charge une partie de l'œuvre. On pourrait imaginer ici peut-être jusqu'à six copistes différents, correspondant à des groupes des manuscrits d'origine.

Le contexte de translittération du manuscrit original nous échappe totalement. Il est toutefois intéressant de constater la période probable à laquelle on réalise la copie. On pense bien évidemment à Michel Psellos, grand personnage souvent proche de l'empereur entre 1042 et sa mort 1078. C'est également un érudit, auteur de nombreux ouvrages, et grand admirateur de l'Antiquité. Sa personnalité serait toute indiquée pour mettre en œuvre un travail d'une telle ampleur, d'autant que le manuscrit était vraisemblablement conservé au palais impérial. Nous aurions tendance à également rapprocher le moment de la copie de celle qui est faite, à la demande du césar Jean Doukas, du De Administrando Imperio (cf. supra § 3.1), dont le manuscrit original était peut-être conservé au même endroit.

\subsubsection{La réalisation d'un premier abrégé}

Par la suite, le texte de Stéphane de Byzance est l'objet d'une première épitomisation (H1, correspondant à un manuscrit que nous appellerons $[\beta]$ ), qui n'a rien à voir avec celle d'Hermolaos et que nous attribuerons, à défaut de connaître son auteur, à un pseudoHermolaos.

112 Il est important de vérifier si cet abrégé a bien été fait sur le manuscrit $\mathrm{S}$. Pour cela, il est possible d'analyser les erreurs communes aux quatre manuscrits principaux de la partie 
correspondante du livre XIII et de les comparer avec celles que nous avons détaillées cidessus du manuscrit du XI $\mathrm{e}^{\mathrm{e}}$ siècle.

Bien que le nombre de fautes communes aux quatre manuscrits pour cette partie du livre XIII soit assez faible, on constate que plusieurs erreurs dérivent de $S(\delta 142,5 ; \delta 144$,

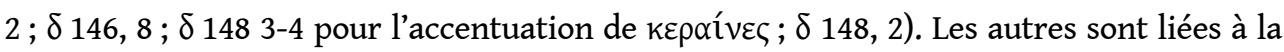
double abréviation du texte. Malgré lle nombre réduit d'erreurs, il est très vraisemblable que l'archétype de nos manuscrits dérive de $\mathrm{S}$. C'est l'hypothèse que nous retiendrons pour la suite de notre étude.

114 Pour renforcer l'hypothèse de cette filiation, il est possible d'étudier également les fautes des livres qui relèvent de la seule abréviation du pseudo-Hermolaos (livres I à III et LXVIII à LX).

115 La quasi-totalité des fautes peut s'expliquer par des erreurs d'onciales, liées à la translittération du manuscrit. L'abréviation rajoute quelques fautes, mais relativement peu. Le premier abrégé est donc encore révélateur du manuscrit primitif et doit certainement avoir été réalisé d'après la translittération du $\mathrm{XI}^{\mathrm{e}}$ siècle.

116 La perte de l'archétype de nos manuscrits ne permet pas de savoir précisément la forme du manuscrit de l'abrégé. Nous avons cependant le nombre de caractères des trois premiers livres (55 785 caractères). Les différents manuscrits que nous connaissons ont majoritairement 30 lignes. Si l'on prend un manuscrit du même type, avec environ 39 caractères par ligne, on arrive à 1170 caractères par page soit environ 47-48 pages. Il est probable que l'on avait un nombre entier de cahiers (très vraisemblablement des quaternions) c'est pourquoi nous proposerons un total de 48 pages, soit 3 quaternions pour le début de l'abrégé. Dans ce cas, le pinax avait totalement disparu de l'abrégé ; il n'était plus vraiment utile ${ }^{85}$; seule subsistait la mention de changement de livre. Si l'on rapporte le nombre théorique de caractères des trois premiers livres avec un manuscrit ayant une moyenne de 1012 caractères par page, comme le manuscrit de Théophile, on obtient environ 80 folios, soit 10 quaternions.

117 Pour les trois derniers livres on a 29833 caractères; si l'on conserve le nombre de 39 caractères par ligne pour 30 lignes par page, cela donne environ 25-26 pages ; il s'agit probablement de 13 folios soit 2 quaternions dont les derniers folios n'auraient pas été écrits. Si l'on prend l'hypothèse à environ 1012 caractères par page, on obtient 29-30 pages, soit quinze folios (deux quaternions dont le dernier folio serait vierge). Les trois derniers livres sont donc presque deux fois plus courts que les trois premiers, du fait d'un nombre moins important de notice et d'information grammaticale par livre.

$118 \mathrm{Au}$ total, c'est peut-être un manuscrit réduit d'un tiers par rapport à l'édition du XI siècle, soit entre 400 et 490 folios, suivant le nombre de caractères par page (de 1010 à 1 170). Il faut noter enfin que nous n'avons plus aucune mention dérivant du manuscrit d'origine, alors même que nous savons qu'il subsistait au moins partiellement. Il n'a quoi qu'il en soit pas été détruit après l'épitomisation.

119 Nous ne connaissons pas la date exacte de la rédaction du premier abrégé, mais nous pouvons la caler chronologiquement entre la copie de Théophile et le moment où Eustathe de Thessalonique rédige ses divers commentaires (comme nous le verrons plus loin, celui-ci indique expressément qu'il utilise l'épitomé de Stéphane de Byzance). Nous réduirons ce laps de temps par l'utilisation probable de ce premier abrégé par le lexicographe Syméon (cf. infra). C'est pourquoi nous proposons le début du XII siècle pour sa rédaction. 
Cet épitomé était certainement plus aisé à consulter que l'œuvre d'origine ${ }^{86}:$ du fait d'une réduction moyenne d'environ deux-tiers du texte de Stéphane de Byzance (cf. supra § 2.4), il opérait probablement un regroupement de trois livres primitifs en un seul. La déconnexion entre cet abrégé et celui d'Hermolaos permet d'expliquer comment ce texte a été transmis jusqu'à Eustathe de Thessalonique sans jamais apparaître avant le $\mathrm{XII}^{\mathrm{e}}$ siècle, ce qui semble impossible si l'abrégé datait effectivement au $\mathrm{VI}^{\mathrm{e}}$ siècle.

121 L'abrégé a été fait de façon totalement anonyme, en conservant la dénomination de Stéphane de Byzance comme il est d'usage pour les épitomés. Son auteur était familier avec les principes de l'accentuation. Ainsi, de nombreuses erreurs d'accentuation de la copie de Théophile ont été corrigées et n'apparaissent plus ensuite.

Le manuscrit [ $\beta]$ a mis à la disposition des érudits les Ethnika. Il a ainsi servi pour la rédaction de plusieurs lexiques et, dans la seconde moitié $d u$ XII ${ }^{e}$ siècle, à Jean Tzetzès et Eustathe de Thessalonique ${ }^{87}$. Il s'agit très probablement d'un travail qui s'est avéré nécessaire pour un usage de type lexicographique, très en vogue au cours des $\mathrm{XII}^{\mathrm{e}}$ et XIII siècles.

\subsubsection{L'Etymologicum Symeonis et l'Etymologicum Magnum}

Les lexiques d'époque byzantine sont nombreux et n'ont pas tous été publiés ni étudiés. Ils représentent une source importante pour des auteurs depuis disparus, c'est pourquoi ils sont examinés depuis plus d'un siècle de manière attentive. Nous nous baserons pour leur étude sur le travail de F. Schironi (2004), qui a analysé les fragments de l'œuvre d'Aristarque dans ces Etymologica. Compte tenu du manque d'édition intégrale de ces lexiques, il n'a pas été possible de mener un travail complet sur l'utilisation de Stéphane de Byzance ${ }^{88}$.

L'un d'entre eux, appelé Etymologicum Symeonis, du nom du grammairien qui l'a composé89 , est particulièrement important pour notre propos. Rédigé dans la première moitié du XII ${ }^{\mathrm{e}}$ siècle ${ }^{90}$, il prend pour source principale l'Etymologicum Genuinum, une compilation probablement réalisée à Constantinople dans l'entourage de Photios, au milieu du $\mathrm{IX}^{\mathrm{e}}$ siècle $^{91}$, et qui a surtout utilisé le lexique composé par Oros et celui de Methodios ${ }^{92}$. L' Etymologicum Symeonis complète ce texte par un certain nombre de sources extérieures dont Stéphane de Byzance. Il est le premier à utiliser cet auteur; c'est par son intermédiaire que l'on en retrouve des mentions dans l'Etymologicum Magnum, autre compilation mieux connue du milieu du XII ${ }^{\mathrm{e}}$ siècle.

On a conservé pour ce lexique deux manuscrits, présentant des textes différents: le

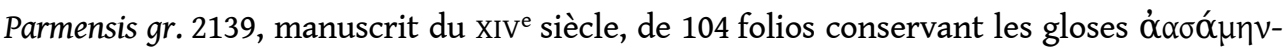

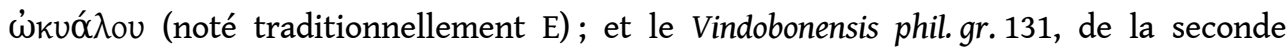

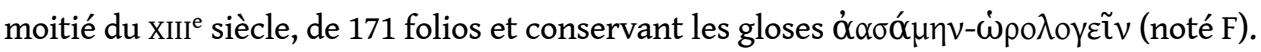

L'Etymologicum Symeonis présente des notices assez brèves mais il semble à travers le corpus que nous avons pu examiner qu'il utilise le texte du premier abrégé, qui semble largement suffisant pour un lexique général. Dans les exemples que donne Reitzenstein, celui du lemme Anaguroûs est assez révélateur : le texte est quasi semblable à celui que nous possédons. Cette notice est la première du livre $\mathrm{V}$, conservé dans la seconde phase d'abréviation mais qui est ici assez proche du premier abrégé. Il est donc très certainement basé sur le manuscrit [ $\beta]$. Toutes les mentions que nous avons pu examiner confortent cette hypothèse. 
Ce lexique est le premier à reprendre largement la matière de Stéphane de Byzance, ce qui implique une sorte de redécouverte de l'importance de ce texte pour les lexiques; de nombreuses notices proviennent exclusivement des Ethnika ${ }^{93}$. Comme l'abrégé de Stéphane de Byzance peut avoir été réalisé au même moment, on notera que cela constitue une coïncidence peut-être significative.

Vers le milieu du XII ${ }^{\mathrm{e}}$ siècle, l'Etymologicum Magnum reprend les notices déjà rédigées et les complète avec d'autres compilations, sans retourner aux différentes sources antiques qu'il mentionne (BERGER, 1972: XIX-XXIII) ${ }^{94}$. Ce lexique réutilise très largement l' Etymologicum Symeonis, à tel point qu'il a pendant longtemps été considéré comme plus ancien. Il n'est pas certain que l'auteur soit retourné au texte de l'abrégé de Stéphane de Byzance. Il est utilisé par Eustathe de Thessalonique, ce qui permet de donner un terminus ante quem à sa rédaction.

Contrairement à ce que proposaient F. LASSERRE et N. LIVADARAS (1976: XV-XVI), F. SCHIRONI (2004: 18-19) et M. BILLERBECK et alii (2006: 32*) ${ }^{95}$ distinguent l'Etymologicum

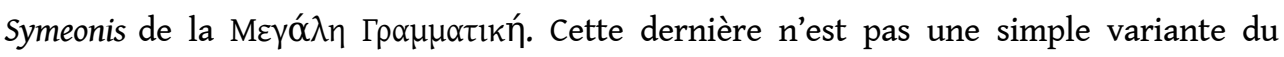
premier mais bien une amplification, utilisant également l'Etymologicum Magnum (et donc nettement postérieure). Deux manuscrits de cette recension sont connus : le Laurentianus S. Marci 303 (lettre C, de parchemin, daté de 1291) et le Vossianus gr. 20 (lettre F, parchemin, datable également de la fin du XIII ${ }^{\mathrm{e}}$ siècle). Elle ne sera pas utile pour notre propos.

\subsection{La seconde moitié du XII ${ }^{\mathrm{e}}$ siècle}

Deux auteurs et érudits majeurs de la seconde moitié du XIII siècle ont utilisé le lexique de Stéphane de Byzance : Jean Tzetzès et Eustathe de Thessalonique.

\subsubsection{Jean Tzetzès}

On considère généralement que le dernier à avoir lu et utilisé Stéphane de Byzance est Jean Tzetzès (dans ses Chiliades [III, v. 818-820] entre 1144 et 1170) qui cite son œuvre avec

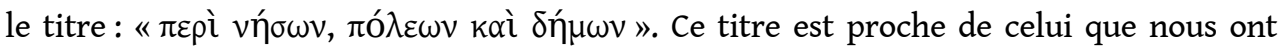
conservé certains des manuscrits du pseudo-Hermolaos. Il n'est donc pas impossible que le poète ait simplement signalé le nom de l'œuvre qu'il avait sous les yeux, comme a pu le faire Eustathe de Thessalonique. Rien ne prouve qu'il s'agisse de l'œuvre intégrale; l'abrégé, ayant probablement été diffusé, a très bien pu servir de source à Jean Tzetzès.

\subsubsection{Eustathe de Thessalonique}

Dans le $3^{\mathrm{e}}$ quart du XII ${ }^{\mathrm{e}}$ siècle, Eustathe de Thessalonique (1115-1195/6) (coHN, 1907) a composé plusieurs commentaires sur Homère (Iliade et Odyssée) et sur Denys le Périégète avant de devenir évêque de Thessalonique en $1175^{96}$. Il utilise très largement l'abrégé de

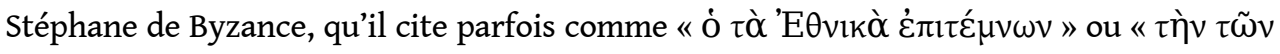
'E $\theta v i \kappa \tilde{\omega} v \dot{\varepsilon} \pi ı \tau o \mu \eta ́ v »^{97}$. Il est le premier à faire référence de façon explicite à un abrégé de l'œuvre de Stéphane de Byzance. Nous sommes donc sûrs qu'il a eu en main au moins la version du pseudo-Hermolaos. Il est probable, comme à son habitude, qu'il a possédé une copie de cet abrégé que nous appellerons [ $\left.\beta^{\prime}\right]$. Les mentions qu'il en fait (en particulier dans le commentaire sur l'Iliade ou dans celui de Denys le Périégète), sont importantes 
pour comprendre quel texte il avait sous les yeux; c'est pourquoi nous avons comparé une partie des mentions fournies par Eustathe avec les notices de notre Épitomés ${ }^{98}$. Quand l'on compare le texte des notices conservées avec ses propres mentions, on comprend la façon dont Eustathe utilise le texte de Stéphane de Byzance, très différente de celle d'un Constantin Porphyrogénète. Ici, l'ordre du texte d'origine est fonction de la reformulation qu'opère Eustathe: les phrases d'origine sont réutilisées souvent dans le désordre; l'auteur ne cite pas des notices complètes, il les intègre dans son discours. Cela rend la comparaison difficile et surtout la restitution du texte d'origine plus aléatoire.

Malgré ces difficultés, on s'aperçoit que le texte que lisait Eustathe n'est pas celui de la dernière étape d'abréviation ${ }^{99}$. Par exemple, la notice Mariandunia, conservée seulement dans un livre résumé, n'a pas la mention que signale Eustathe à propos de l'ethnique :

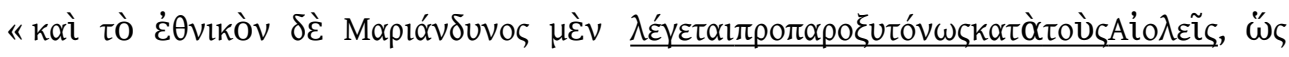

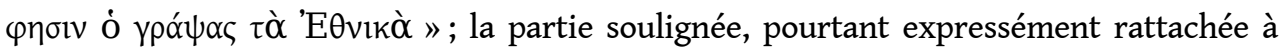
Stéphane de Byzance, a disparu du résumé.

134 La question de savoir si Eustathe lisait l'épitomé du pseudo-Hermolaos ou le texte du second abréviateur est plus complexe. Les mentions qui proviennent des livres II et III témoignent d'une coïncidence très forte avec le texte du pseudo-Hermolaos ${ }^{100}$. Si l'on enlève les livres qui ont été résumés, il reste un certain nombre de mentions pouvant être comparées avec la seconde abréviation. Dans ce cas, quelques indications d'Eustathe à propos de textes provenant des Ethnika semblent se rapporter à des passages que nous n'avons plus; on le constate par exemple pour la notice Ausôn, avec une référence à Calypso pourtant attribuée explicitement à notre texte, ou pour la notice Hêlis. Bien sûr, la seconde abréviation peut donner un texte finalement assez proche de celui du pseudoHermolaos une fois que l'on enlève les citations et les formes annexes, qui n'intéressent pas Eustathe dans ses démonstrations. On peut comprendre que l'on ait cru jusqu'à présent que l'Épitomé que nous avons conservé était celui que lisait Eustathe ${ }^{101}$.

\subsubsection{Une glose d'Eustathe de Thessalonique dans l'Épitomé ?}

La notice Tamiathis fait référence à une des œuvres de Georgios Chœroboscos, l'

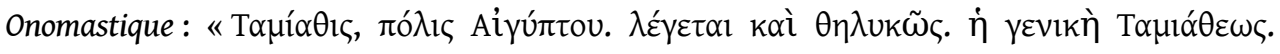

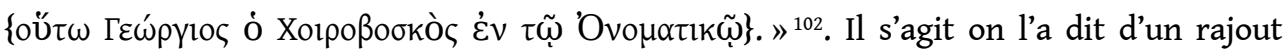
postérieur, qui doit provenir d'un lexicographe.

Cette manière de citer Chœroboscos se retrouve à plusieurs reprises chez le pseudo-

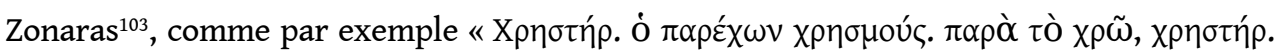

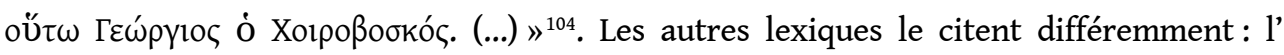

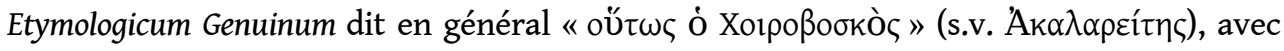
parfois la mention de l'œuvre mais toujours introduite par « $\varepsilon \dot{i} \zeta »^{105}$; on trouve aussi

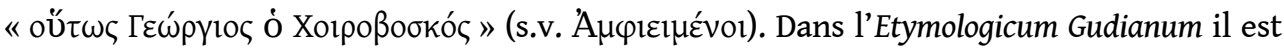

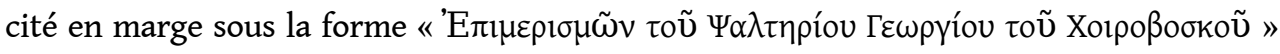
(s.v. Apó) mais la plupart du temps sans nom d'œuvre. L'Etymologicum Symeonis indique

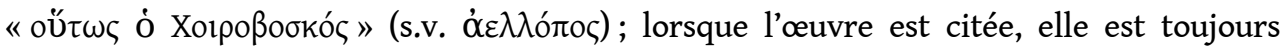

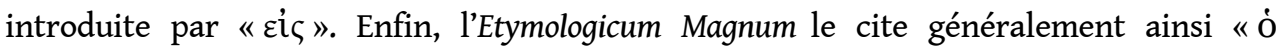

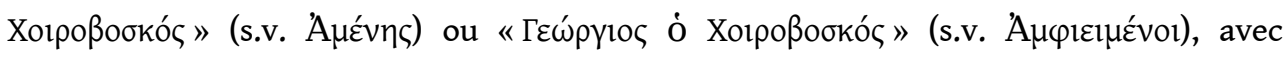

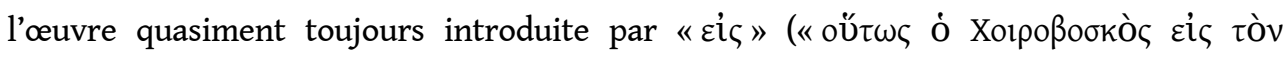

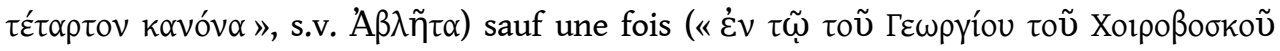

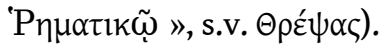




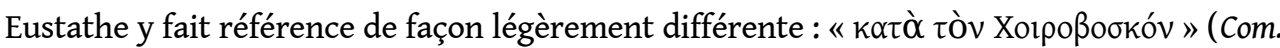

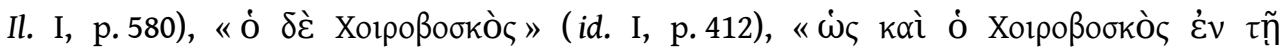

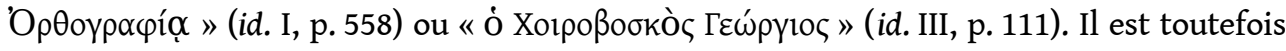
le seul avec l'Etymologicum Magnum à introduire l'œuvre par ćv.

S'il est difficile de choisir entre les lexicographes ou Eustathe, il est troublant de constater que la mention inscrite sur le manuscrit de l'abrégé peut correspondre à sa façon de travailler. On peut mettre cette glose en parallèle avec celles de la Souda, signalées cidessus, qu'il a rédigées sur le manuscrit. La manière d'introduire le nom de l'œuvre, typique d'Eustathe, rend vraisemblable l'attribution à cet auteur, comme le pensait déjà A. Diller ${ }^{106}$, même s'il n'est pas possible de trancher de façon définitive.

Il est probable que le manuscrit intermédiaire [ $\left.\beta^{\prime}\right]$, copié pour Eustathe de Thessalonique ait été annoté par Eustathe lui-même et qu'il soit à l'origine de l'archétype de nos manuscrits.

Eustathe de Thessalonique utilisant l'épitomé du pseudo-Hermolaos [ $\beta]$, le texte du second abréviateur a toutes les chances d'être postérieur à la seconde moitié $d u \mathrm{XII}^{\mathrm{e}}$ siècle. Les lacunes que l'on connaît maintenant n'existaient pas au XII ${ }^{\mathrm{e}}$ siècle, comme en témoigne l'utilisation par Eustathe de Thessalonique des notices Kíkovę et

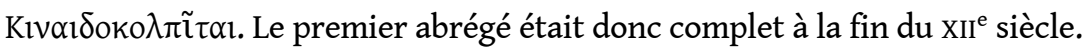

\subsection{De la fin du XII à la fin du XIV siècle}

141 Après la période de floraison du XII ${ }^{\mathrm{e}}$ siècle, et la prise de Constantinople par les Croisés en 1204, Stéphane de Byzance retombe dans l'oubli. On sent que la survie du texte abrégé est difficile ${ }^{107}$.

Il est probable que le manuscrit d'origine ait été dispersé lors du siège de Constantinople. Ce serait donc un hasard de conservation qui aurait permi à un quaternion d'un des volumes de la translittération $\mathrm{du} \mathrm{XI}^{\mathrm{e}}$ siècle de parvenir jusqu'à nous.

\subsubsection{Le Lexique du pseudo-Zonaras}

143 Le lexique du pseudo-Zonaras, appelé également Lexicon Tittmanianum, du nom de son éditeur, est composé à la charnière des $\mathrm{XII}^{\mathrm{e}}$ et $\mathrm{XIII}^{\mathrm{e}}$ siècles. Il est basé sur l'Etymologicum Symeonis et donne un texte plus complet que les manuscrits E et F. Il a fait l'objet d'une publication certes ancienne (TITTMAN, 1818-1820) mais érudite et toujours utilisable. De très nombreux lemmes proviennent de Stéphane de Byzance. Il nous semble probable que ceux-ci viennent d'une nouvelle compilation depuis l'abrégé et non directement de l' Etymologicum Symeonis; toutefois, les preuves nous manquent. Du fait de l'absence de publication totale de l'Etymologicum Symeonis, c'est le pseudo-Zonaras qui semble avoir le plus emprunté à Stéphane de Byzance (BILLERBECK et alii,2006:31-33*), mais il n'est probablement que le dernier maillon d'une chaîne. Il utilise vraisemblablement toujours l'abrégé du pseudo-Hermolaos.

\subsubsection{La seconde abréviation}

C'est peut-être dans le courant du XIII $^{\mathrm{e}}$ siècle qu'a lieue la seconde abréviation $\mathrm{H} 2$ (manuscrit $[\gamma]$ ), ne conservant qu'environ $60 \%$ du texte du pseudo-Hermolaos, soit une 
réduction à environ $20 \%$ du texte d'origine. Il est très probable que cette abréviation a concerné l'ensemble du premier épitomé, bien que nous n'en ayons conservé qu'une partie. La mention interpolative de Georgios Choroeboscos pourrait alors avoir été intégrée dans cette seconde phase de réduction du texte, ce qui témoignerait d'une rédaction sur la base de l'exemplaire d'Eustathe de Thessalonique [ $\left.\beta^{\prime}\right]$. Nous savons que plusieurs de ses manuscrits sont parvenus jusqu'à nous. Il ne serait donc pas étonnant que son exemplaire de Stéphane de Byzance ait servi encore et soit devenu le seul exemplaire accessible.

Le premier abrégé, qui servait de modèle, avait peut-être déjà perdu quelques quaternions, correspondant semble-t-il à des livres entiers (XXIV, XXVIII et XXXVII). Il est vraisemblable que dans ce premier abrégé les livres commençaient par un recto de folio, ce qui ne sera probablement plus le cas à partir du second abrégé.

Cette deuxième phase d'abréviation représenterait un manuscrit d'une taille comprise entre 240 et 300 folios, ce qui devient une épaisseur normale.

L'Épitomé présente une particularité quant à l'usage des sources. Comme nous l'avons dit supra (\$2.3), toutes les mentions d'auteurs qui se trouvaient dans l'œuvre d'origine ont été réduites successivement, avec perte généralement de la citation voire même de la totalité de la mention de l'œuvre et de l'auteur. Or, les mentions de Lycophron sont les seules à avoir conservé quasiment toutes leurs citations : sur 56 notices où cet auteur est encore mentionné, 7 seulement ont perdu leur citation (dont une dans le livre I abrégé par le pseudo-Hermolaos). Aucun autre auteur n'a été traité de cette manière (à l'exception peut-être d'Homère ?), ce qui semble impliquer une attention particulière pour le texte de ce poète. On pense immédiatement au commentaire rédigé par Jean Tzetzès, qui a mis en lumière cet auteur difficile. Il est probable que le second abrégé ait été réalisé peu de temps après, car c'est son auteur qui a surtout fait disparaitre les citations. Il s'agit là d'un choix délibéré. Nous nous trouvons probablement ici sous le règne des Paléologues et du renouveau des études sur les textes antiques à cette époque.

\subsubsection{L'utilisation de l'œuvre au XIV siècle}

148 F. LASSERRE (1959: 47), signale une glose du XIV e siècle qui pourrait provenir selon lui de Stéphane de Byzance. Le copiste du Palatinus Heildelbergensis 129 (probablement Nicéphore Grégoras $^{108}$ pour le texte qui nous intéresse) donne une notice sur la ville d'Assos:

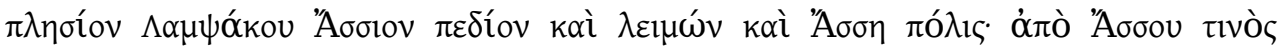

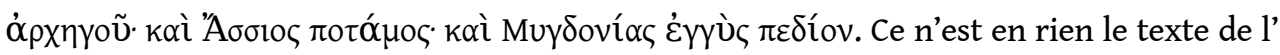
Épitomé ${ }^{109}$ et il semble difficile d'y voir un texte issu de l'œuvre originale. Nous ne savons pas de quel lexique ceci provient, peut-être de celui de Diogenianus?

149 Nous avons peut-être un repère chronologique avec une mention dans le manuscrit Monacensis gr. 380 ; si l'on en croit M. BILLERBECK et alii (2006: $\left.35^{*}\right)$, il conviendrait de la dater du XIV siècle. Ce manuscrit de 568 folios contient de très nombreux textes religieux ${ }^{110}$. Au folio 533, le rédacteur des Quaestiones Canonicae de Nicolas Mystikos cite plusieurs auteurs anciens pour le genre de certains noms géographiques ${ }^{111}$. Il donne trois

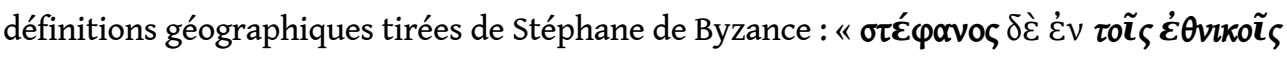

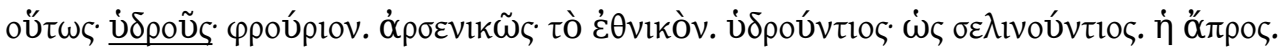

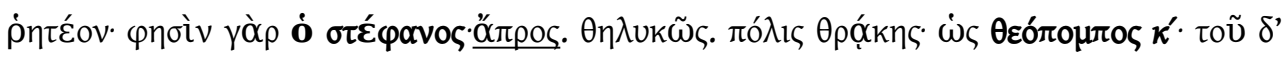

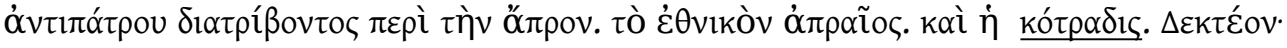

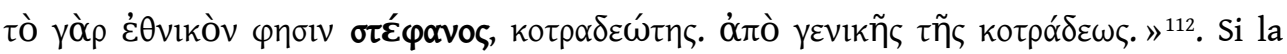


mention reprend de manière précise le texte de Stéphane de Byzance que le copiste avait sous les yeux, cela pourrait être une indication du niveau d'abréviation. Pour les trois lemmes, le texte de l'Épitomé ${ }^{113}$ est celui de la seconde abréviation; or, le texte du manuscrit s'apparente beaucoup plus au résumé qu'à la notice de l'épitomé.

La datation même du manuscrit n'est pas certaine. Si M. Billerbeck, à la suite de HARDT (1810 : 131) indique le XIV ${ }^{\mathrm{e}}$ siècle, J. DARRouzès (1981 : notice $\mathrm{n}^{\circ} 437$ ), dans son étude des notitiae episcopatum ecclesiae Constantinopolitae, propose les $\mathrm{XII}^{\mathrm{e}}$-XIII ${ }^{\mathrm{e}}$ siècles. Il nous semble difficile toutefois de placer ces mentions avant le XIV siècle, si l'on est bien en présence du résumé. Nous n'avons pas vu le manuscrit, mais une note marginale peut quoi qu'il en soit être nettement postérieure au texte lui-même.

M. Billerbeck porte également l'attention sur un fragment de lexique (gloses ỏ $\alpha \sigma \alpha ́ \mu \eta \eta-$ $\dot{\alpha} \lambda \varepsilon \omega ́ \mu \varepsilon \theta \alpha)$, appelé Etymologicum Casulanum et conservé aux folios 167-174 du Vaticanus gr. 1276. Ce manuscrit, écrit à San Nicola di Casole au XIv siècle $^{114}$, fait partie d'un ensemble de 70 manuscrits (Vaticanus gr. 1218-1287) ayant appartenu au cardinal Carafa (1538-1591), abbé commandaire du monastère. Il comprend de très nombreux textes religieux et quelques textes différents comme le De Tropis du grammairien Tryphon (fol. 83-89v)ou le De natura hominum d'Hippocrate. Il semble bien qu'il reproduise toute une série d'œuvres du XIII ${ }^{\mathrm{e}}$ siècle " composées en Italie méridionale ou qui suscitent la curiosité des lettrés locaux : poésie et œuvres grammaticales » (DARROUŻ̀s, 1970: 222. Voir également p. 221, n. 3).

L'Etymologicum Casulanum a été rédigé en compilant plusieurs sources, dont l'Etymologicum Genuinum (dans sa recension du manuscrit A, Vaticanus gr.1818) et l'Etymologicum Gudianum, rédigé en Italie $\mathrm{du}$ Sud dans le courant $\mathrm{du} \mathrm{XI}^{\mathrm{e}}$ siècle, et dont on conservé l'archétype ${ }^{115}$. Le manuscrit A de l'Etymologicum Genuinum, avec ses gloses marginales tirées de l'Etymologicum Gudianum, était alors conservé dans le monastère de Casole ${ }^{116}$. L' Etymologicum Casulanum, édité par O. PARLANGÈLI (1953), a été compris comme une variante de l'Etymologicum Symeonis par F. LASSERRE et N. LIVADARAS (1976) et porte, dans leur publication, la lettre $\mathrm{c}$ dans la liste des manuscrits.

Le texte de notre Épitomé , pour les gloses de l'Etymologicum Casulanum, correspond à une partie peu abrégée du texte, mais il n'est pas possible de savoir sur quelle phase d'abréviation la glose s'est effectuée. Il est cependant important de noter la probable présence, dans le sud de l'Italie, d'un épitomé de Stéphane de Byzance dans le courant du $\mathrm{XIV}^{\mathrm{e}}$ siècle, qui pourrait être à l'origine de l'archétype de nos manuscrits.

\subsubsection{Le résumé, dernière étape de l'abréviation}

154 Le second épitomé a à son tour été résumé (manuscrit [8]) d'une manière telle qu'il ne reste quasiment plus rien du texte d'origine (en moyenne environ $11 \%$ du texte conservé, soit une réduction d'environ $55 \%$ de la seconde abréviation). Il s'agit d'une phase probablement tardive, pour laquelle nous n'avons aucune datation envisageable, ni aucun lien avec quelque auteur byzantin tardif. Pour distinguer cette dernière phase, la plus radicale pour les informations contenues, nous parlerons de résumé dans la suite de notre propos.

155 Dans notre Épitomé, le résumé concerne surtout les notices comprises entre Patukos et Surapolêttos. Le texte n'a conservé que peu d'information et s'apparente à un index un peu développé. Il n'est pas possible de savoir si à l'origine l'ensemble de l'œuvre avait fait 
l'objet d'un résumé ; c'est toutefois probable et c'est l'hypothèse que nous adopterons. Avec ce travail, on est toujours dans un contexte lexicographique qui tend à se réduire au fur et à mesure que l'on abrège les lexiques (on le voit par exemple pour les manuscrits de certains etymologica datés du XIV ${ }^{\mathrm{e}}$ siècle.

D'après la taille du texte d'origine, le manuscrit de ce résumé devait cependant faire encore entre 130 et 165 folios.

\subsection{Les $\mathrm{XV}^{\mathrm{e}}$ et $\mathrm{XV} \mathrm{l}^{\mathrm{e}}$ siècles}

L'histoire du texte de Stéphane de Byzance est connue de façon certaine à partir de la fin du $\mathrm{Xv}^{\mathrm{e}}$ siècle. Il n'est pas impossible qu'il faille placer durant ce siècle la réalisation du résumé ; les marqueurs chronologiques sont malheureusement totalement absents.

\subsubsection{L'archétype des manuscrits conservés}

C'est à une date elle aussi tardive qu'intervient l'archétype [ $\omega]$ des manuscrits conservés. Il mélange en fait trois phases d'abréviation, probablement des morceaux de leurs manuscrits. Il est très probable que cet archétype est très tardif (probablement le courant du Xv $v^{e}$ siècle, peut-être après la chute de Byzance). Nous ne savons pas d'ailleurs s'il s'agit d'un manuscrit composite, ayant rassemblé plusieurs fragments de différentes versions ou si l'ensemble a fait l'objet d'une copie homogène. Les variantes pour le début de la

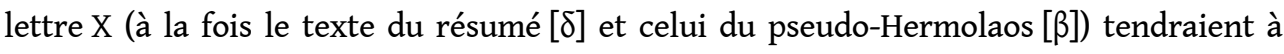
privilégier la première hypothèse. Quoiqu'il en soit, le copiste avait sous les yeux un manuscrit du pseudo-Hermolaos très lacunaire, probablement celui provenant d'Eustathe de Thessalonique [ $\beta$ '] qui comprenait les livres I-III et LVIII-LX de Stéphane de Byzance. Il l'a complété par le second abrégé $[\gamma]$. Ce manuscrit était également fortement incomplet : outre les lacunes des livres XXIV, XXVIII et XXXVII, il manquait probablement les livres XIV, XXIX-XXX, XXXV et XL à L. L'auteur du manuscrit a compensé ces lacunes autant que possible par le résumé $[\delta]$.

La numérotation des livres est également révélatrice de cet assemblage. Les livres provenant de $[\delta]$ ne sont jamais signalés, sauf pour le livre XIV, facilement restituable puisque coincé entre deux livres conservés et numérotés chez $[\gamma]^{117}$. Les dix derniers livres n'ont pas conservé de numérotation; enfin, les livres XXIII, XXVI, XXVII, XXXVIII et XXXIX, pourtant repris de $[\gamma]$, n'ont pas conservé de trace de numérotation de livre.

Par ailleurs, il faut noter que jamais le manuscrit original de Stéphane de Byzance [S] qui pouvait encore exister à cette époque n'a été utilisé pour notre archétype ; il n'était pas conservé au même endroit et est resté inutilisé jusqu'à sa redécouverte à l'époque Moderne.

\subsubsection{La tradition manuscrite}

161 Nous ne nous étendrons pas sur les aspects philologiques ni sur la tradition manuscrite de l'Épitomé, qui nous éloigneraient ici de notre étude. La tradition des manuscrits que nous avons conservés a été traitée de façon particulièrement développée par A. DILLER (1938: 339-348) repris et complété par M. BILLERBECK et alii (2006: 8-29*) pour sa nouvelle édition de l'œuvre. Notons cependant que tous les manuscrits que nous connaissons de l'Épitomé datent au mieux de la fin du $\mathrm{xv}^{\mathrm{e}}$ siècle ${ }^{118}$ et constituent la copie d'un même manuscrit 
conservé dans la bibliothèque de Jean Calphurnius (MARCOTTE, 1987 : 193 ; MARCOTTE, 2000 : 265-266). Celui-ci, éditeur puis récipiendaire de la chaire de rhétorique au Studio de Padoue à partir de 1486, accueille en 1491 Janos Lascaris en route pour la Grèce pour acquérir des manuscrits grecs ; le voyageur voit ainsi dans la bibliothèque de son hôte le manuscrit de l'Épitomé de Stéphane de Byzance et le signale ${ }^{119}$. Ce manuscrit est mentionné à nouveau le 18 janvier 1503, lors de l'établissement de la première liste du catalogue de la bibliothèque de Calphurnius, qui semble avoir été léguée au couvent de San Giovanni di Verdara à Padoue ${ }^{120}$. Il n'a donc pas disparu au moment de l'editio princeps d'Aldus Manutius en $1502^{121}$, mais nul ne sait ce qu'il devient ensuite. Il n'apparaît pas dans le catalogue rédigé au XVII ${ }^{\mathrm{e}}$ siècle.

\subsection{Stemma de la transmission de l'œuvre}

Pour résumer les acquis de notre étude, nous avons dressé un stemma de la tradition manuscrite sous deux formes. La première (fig. 7) est celle que l'on trouve de façon classique dans toute édition de texte ancien. Nous avons complété celui que donne $\mathrm{M}$. BILLERBECK et alii $\left(2006: 29^{*}\right)$ par les hypothèses de transmission que nous avons développées ci-dessus. Les lecteurs du manuscrit apparaissent à droite, en italique.

Le second stemma (fig. 8) est tracé en utilisant le principe des diagrammes stratigraphiques que dressent les archéologues. Il permet de mettre en évidence la pérennité de certains manuscrits malgré leur copie, et intègre donc une indication de durée pour les manuscrits. Cette information peut être importante ; on ne la trouve pas dans le premier stemma où n'apparaissent que les transmissions. Nous pouvons ainsi mieux faire apparaitre la disparition de certains manuscrits.

Comme pour les diagrammes stratigraphiques archéologiques, l'important est de noter ici les liens de relations (antérieur/postérieur à) mais non de garder une échelle de temps homogène. Ceci explique la dilatation de certaines périodes au sein du diagramme. Nous partons de la période la plus ancienne, à la base du diagramme, pour arriver à notre époque. En haut du diagramme sont mentionnés les manuscrits (et l'édition d'Aldus) dont nous disposons. Il est ainsi facile d'arriver à leur origine.

La double (voire triple) paternité est également plus facile à rendre sous cette forme. 


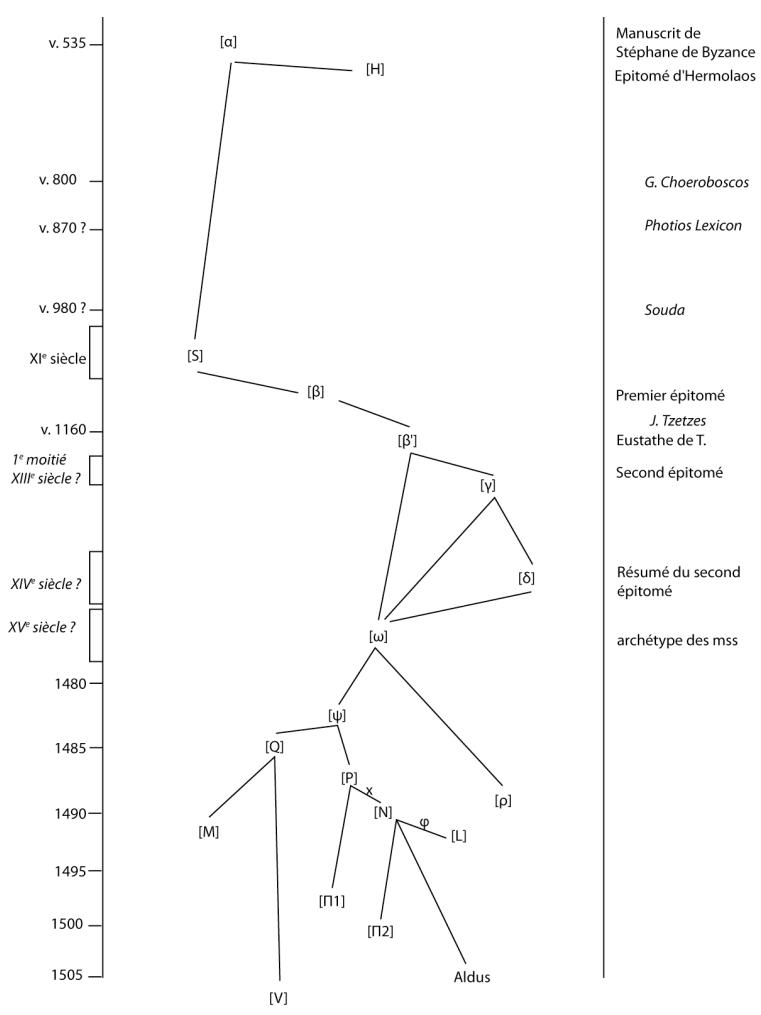

Fig. 7. Stemma traditionnel de la tradition manuscrite (d'après BILLERBECK et alii, 2006 : 29* pour la partie basse).

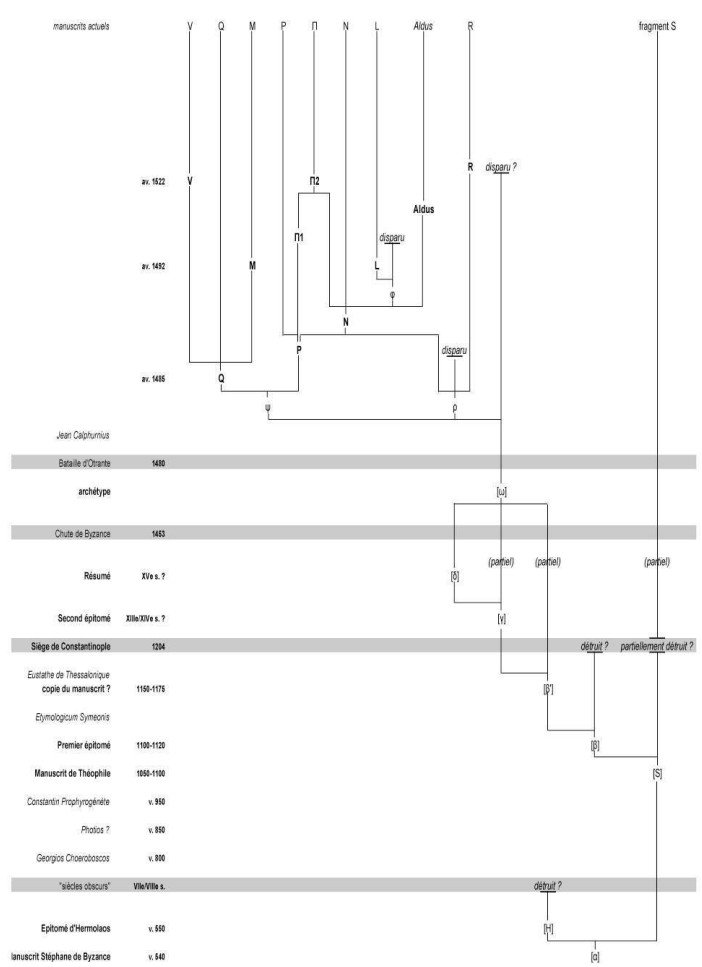

Fig. 8. Diagramme chronologique de la tradition manuscrite (d'après BILLERBECK et alii, 2006 : 29* pour la partie basse). 


\section{BIBLIOGRAPHIE}

Acconcia Longo Augusta, JACOB André, « Une anthologie salentine du XIV ${ }^{e}$ siècle : le Vaticanus gr. 1276 », Rivista di Studi Bizantini e Neoellenici, n.s. 17-19 (1980-82), p. 149-228.

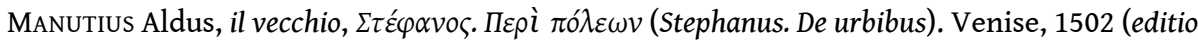
princeps).

BERGER Günter, Etymologicum Genuinum et Etymologicum Symeonis ( $\beta$ ), « Beiträge zur Klassischen Philologie, 45 », Meisenheim am Glan : Verlag Anton Hain, 1972, XXXVIII-188 p.

BILleRbeCK Margarethe, GAertner Jan Felix, Wyss Beatrice, Zubler Christian (éd.), Stephani Byzantii Ethnica : Einleitung, kritischer Text, Ubersetzung und Anmerkungen, « Corpus Fontium Historiae Byzantinae 43/1 et 2 », I : Alpha - Gamma. Berlin/New York : Walter de Gruyter, 2006, X-64*-441 p. ; II : Delta - Iota. Berlin/New York : Walter de Gruyter, 2011, IX-17*-310 p. BURY J. B., The treatiseDe administrando imperio, Byzantinische Zeitschrift, 15, 1906, p. 517-573.

CAVALLO G., Ricerche sulla maiuscula biblica, Florence, 1967.

CoHN Leopold, « Eustathios 18 », Pauly-Wissowa, Realencyclopädie der classischen Altertumswissenschaft, VI, 9, 1907, col. 1452-1489.

DARROUZES Jean, « Un faux acte attribué au patriarche Nicolas (III) », Revue des études byzantines, 28,1970, p. 221-237.

DARROUZES Jean, Notitia episcopatuum ecclesiae constantinopolitanae. Texte critique, introduction et notes . Paris, 1981.

DEVREESSE Robert, Catalogue des manuscrits grecs. Le fonds Coislin, vol. II, Paris : Bibliothèque Nationale, 1945, $428 \mathrm{p}$.

DICKEY Eleanor, Ancient Greek Scholarship. A Guide to Finding, Reading, and Understanding Scholia, Commentaries, Lexica, and Grammatical Treatises, from Their Beginnings to the Byzantine Period. Oxford : University Press, 2007, 345 p.

DILLER Aubrey, "The Tradition of Stephanus Byzantius ", Transactions and Proceedings of the American Philological Association, LXIX, 1938, p. 333-348.

Diller Aubrey, « Excerpts from Strabo and Stephanus in Byzantine Chronicles ", Transactions and Proceedings of the American Philological Association, LXXXI, 1950, p. 241-253.

DILLER Aubrey, The textual tradition of Strabo's Geography. With appendix: The Manuscripts of Eustathius' Commentary on Dionysius Periegetes. Amsterdam, 1975, 222 p.

Doyen-Higuet Anne-Marie, L'Épitomé de la Collection d'hippiatrie grecque. Histoire du texte, édition critique, traduction et notes. Tome I, «Publications de l'Institut orientaliste de Louvain, 54 », Louvain, 2006, $242 \mathrm{p}$.

FRASER P. M., Greek Ethnic Terminology, «A lexicon of Greek personal names, Supplementary volume ", Oxford/New York, 2009, 424 p.

GAISFORD Thomas, Etymologicum Magnum. Oxford, 1848. 
GRoNoviUS J., Fragmentum Stephani Byzantini grammatici de Dodone, cum triplici nupera Latina versione et academicis exercitationibus, Leyde, 1681.

HILGARD A., Prolegomena et scholia in Theodosii Alexandrini canones isagogicos de flexione verborum, « Grammatici Graeci, vol. 4.2 », Leipzig : Teubner, 1894 (repr. Hildesheim : Olms, 1965).

Honigmann Ernst, «Stephanos Byzantios », Pauly-Wissowa, Realencyclopädie der classischen Altertumswissenschaft, III A 2 (1929), col. 2369-99.

HUNGER Herbert, Die hochsprachliche profane Literatur der Byzantiner. Erste Band : Philosophie -

Rhetorik - Epistolographie - Geschichtsschreibung - Geographie, « Handbuch der

Altertumswissenschaft, XII ; Byzantinisches Handbuch, 5.1 », Münich : C.H. Beck'sche

Verlagsbuchhandlung, 1978, XXVI-542 p.

HUNGER Herbert, Die hochsprachliche profane Literatur der Byzantiner. Zwzeite Band: Philologie -

Profandichtung - Musik - Mathematik und Astronomie - Naturwissenschaften - Medizin -

Kriegswissenschaft - Rechtsliteratur, « Handbuch der Altertumswissenschaft, XII ; Byzantinisches Handbuch, $5.2 »$, Münich : Beck, 1978, XX-528 p.

IRIGOIN Jean, Le livre grec des origines à la Renaissance, « Conférences Léopold Delisle », Paris : BNF, 2001, $94 \mathrm{p}$.

Jenkins J. B. \& MoravcsiK G., Constantine Porphyrogenitus. De Administrando Imperio. Washington, 1967.

KALDELlis Anthony, " The Works and Days of Hesychios the Illoustrios of Miletos », Greek, Roman, and Byzantine Studies, 45, 2005, p. 381-403.

KNAUSs Wilhelm, De Stephani Byzantii ethnicorum exemplo Eustathiano. Bonn, 1910.

LASSERRE F., «Etude sur les extraits médiévaux de Strabon », L’Antiquité classique, 28, 1959, p. 32-75.

LASSERRE F. \& LIVARADAS N. (ed.), Etymologicum Magnum genuinum, Symeonis Etymologicum una cum

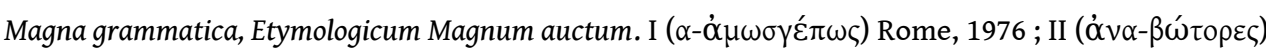
Athènes, 1992.

LEIGHTON Durham \& WILSON Nigel Guy, D'Homère à Erasme, la transmission des classiques grecs et latins. Nouvelle édition revue et augmentée, trad. par C. Bertrand et mise à jour par Pierre Petitmengin. Paris, CNRS, 1988, xvi-262 p.

LEMERLE Paul, Le premier humanisme byzantin. Notes et remarques sur enseignement et culture à Byzance des origines au $x^{e}$ siècle, « Bibliothèque Byzantine, Etudes, 6 », Paris : Presses Universitaires de France, 1971, $326 \mathrm{p}$.

Lounghis T. C., « Sur la date du De Thematibus », Revue des études byzantines, 31, 1973, p. 299-305.

MARCoTTE Didier, « La bibliothèque de Jean Calphurnius », Humanistica Lovaniensia. Journal of NeoLatin Studies, XXXVI, 1987, p. 184-211.

MARCovich Miroslav (éd), Diogenis Laertii Vitae philosophorum. vol. I, Libri I-X. vol. II, Excerpta Byzantina / edidit Miroslav Marcovich. vol. 3, Indices / confecit by Hans Gärtner. « Bibliotheca scriptorum graecorum et romanorum teubneriana », Stuttgart ; Leipzig : Teubner, 1999, 1999 [vol. 1 et 2]. Münich : Leipzig : Saur, 2002 [vol. 3]. 1-826, 346 et x-183 p.

MARGANNE M.-H., Le livre médical dans le monde gréco-romain, « Cahier du CEDOPAL, 3 », Liège, 2004. MEINEKE A. (éd.), Stephani Byzantii Ethnicorum quae supersunt. Berlin, 1849 (réed. Graz 1958, Chicago 1992). 
MonTfAucon Bernard (de), « Fragmentum insigne Stephani Byzantii de urbibus », Bibliotheca Coisliniana, olim Segueriana. Paris, 1715, p. 281-292.

MoRAVCsiK Gyula (éd.), Constantine Porphyrogenitus de administrando imperio, Washington, 2006.

MÜLLER B. A., « Zu Stephanos Byzantios », Hermes, LIII, 4, 1918, p. 337-357.

NERI Camillo \& BILLERBECK Margarethe (ed.), Stephani Byzantii Ethnica. Volumen I : A-G. Corpus Fontium Historiae Byzantinae 43/1. Berlin/New York : Walter de Gruyter, 2006, p. 64*-441. ISBN 978-3-11-017449-6. €148.00, Bryn Mawr Classical Review, 2008.07.64 (http://ccat.sas.upenn.edu/ bmcr/2008/2008-07-64.html).

ODORICO Paolo, «Cadre d'exposition/cadre de pensée - La culture du recueil ». In : VAN DEUn Peter, MACÉ Caroline (éd.), Encyclopedic trends in Byzantium ? Proceedings of the International Conference held in Leuven, 6-8 May 2009, « Orientalia Lovaniensia Analecta, 212 », Louvain/Paris/Walpole, 2011, p. 89-108.

Oнме Heinz, Das Concilium Quinisextum Und Seine Bischofsliste : Studien Zum Konstantinopeler Konzil Von 692. Berlin/New York, Walter de Gruyter, 1990, 423 p.

PARLANGELI Orontius, Il frammento dell'Etymologicum Casulanum nel manoscritto Vat. Gr. 1276, « Bollettino della badia greca di Grottaferrata, 7 », 1953, p. 115-126 et 8, 1954, p. 97-112.

Pertusi A. (éd.), Costantino Porfirogenito, De Thematibus, « Biblioteca Apostolica Vaticana, Studi e testi, 160 », Cité du Vatican, 1952, 210 p.

PonTANi Filippo Maria, Sguardi su Ulisse. Rome : Edizioni di storia e letteratura, « Sussidi eruditi, 63 », $2005,600 \mathrm{p}$.

REITZENSTEIN Richard, Geschichte der grieschischen Etymologika : ein Beitrag zu Geschichte der Philologie in Alexandria und Byzanz, Leipzig, 1897, 408 p.

REYNOLDS Leighton Durham, WILSON Nigel Guy, Scribes and Scholars : A Guide to the Transmission of Greek and Latin Literature, Clarendon Press, 1991, 321 p.

RonConi Filippo, La Traslitterazione dei Testi Greci, « Centro Italiano di studi sull'Alto Medioevo CISAM Quaderni della Rivista di Bizantinistica, 7 », Spoleto, 2003, 201 p.

SMITH Sir William, Dictionnary of Greek and Roman biography and mithology, Londres, 1844-1849, 3 vol.

TENNULIUS [= TEN NUYL] S., Fragmentum de urbibus depromptum ex fidelissimis membranis perantiqui codicis Bibliothecae Seguerianae, Amsterdam, 1669.

Westermann A., Stephani Byzantii 'E $\theta v i k \omega \tilde{v} v$ quae supersunt, Leipzig, 1839.

\section{NOTES}

1. Parmi ces diverses publications, celle d'August Meineke, imprimée en 1849, reste encore à ce jour la seule édition critique complète et a longtemps servi de référence. Depuis 2006, une nouvelle édition est en cours, sous la direction de Margarethe Billerbeck (jusqu'à la lettre iota). Elle est la plus complète d'un point de vue philologique (avec la découverte de nouveaux manuscrits malheureusement aussi tardifs que ceux déjà connus) et fournit la référence aux éditions récentes des fragments d'auteurs perdus que contient le texte; une traduction allemande accompagne cet important travail. 
2. Cet article est issu d'une thèse de doctorat en cours, sous la direction d'Arnaud Zucker (Université de Nice, CEPAM) et Dominique Garcia (Université d'Aix-Marseille, CCJ) ayant pour titre: «l'Épitomé de Stéphane de Byzance comme source historique: l'exemple de l'Europe occidentale ». Il a bénéficié d'une relecture attentive d'Aude Cohen-Skalli (ATER en Histoire grecque à l'Université de Nice-Sophia) et des conseils de Margarethe Billerbeck (professeur de philologie classique à l'Université de Fribourg). Qu'elles en soient ici remerciées.

3. Partie VI, fol. $116 \mathrm{r}-119 \mathrm{bisv}, 120-122 \mathrm{v}$, soit un quaternion en parchemin. Le Coislinianus 228 (appelé $\mathrm{S}$ dans la tradition $\mathrm{du}$ texte) est un manuscrit composite, dont les folios qui nous intéressent sont datés de nos jours du $\mathrm{XI}^{\mathrm{e}}$ siècle (BILLERBECK et alii, $2006: 5^{*}$ ), mais qu'A. DILLER (1938: 334) date $d u X^{e}{ }^{e}$ siècle, sans qu'il en donne la raison. Ce dernier donne en note les différentes éditions de ces folios, qui ont fait l'objet de publications particulières depuis 1669 . Nous y revenons de façon plus détaillé $§ 1.4$.

4. La citation de Stéphane de Byzance concernant cette première notice est incomplète car le manuscrit commence à la ligne 12 de la p. 240 de l'édition de Meineke (BILLERBECK et alii, 2006 : $\left.5^{*}\right)$.

5. Il comprend toutes les entrées entre Eares et Elôros, suivies pour chacune d'un identifiant

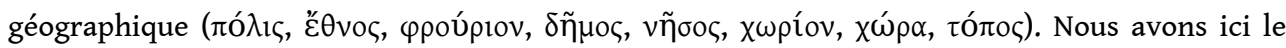
sommaire correspondant à un livre entier de l'ouvrage de Stéphane de Byzance. Le manuscrit indique le livre XI ( $\left(\alpha^{\prime}\right)$ mais dans l'organisation générale de l'ouvrage il s'agit du livre XIV $\left({ }^{\prime} \delta^{\prime}\right)$.

6. Il s'agit très certainement de Constantin Porphyrogénète ; A. Pertusi en doutait.

7. Cf. PLRE, 2, Stephanus Byzantius 24. Voir les testimonia rassemblés dans la nouvelle édition ( BILLERBECK et alii, 2006: 3). Il existe un autre Stéphane de Byzance plus tardif, auteur de commentaires sur l'Art Rhétorique d'Aristote (CRAMER, 1839 : 245 sq), qui pourrait être le même que Stéphane d'Alexandrie (et d'Athènes ?), connu à l'époque d'Héraclius I ${ }^{\mathrm{er}}$.

8. Georgios Chœroboscos, Eis ton onomatikon, 163a. Cf. HonigmanN, 1929 : col. 2374.

9. Com. Il. I, p. 460, 504, 511 et 574 (éd. Van der Valk).

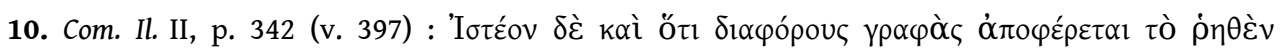

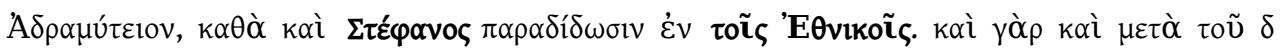

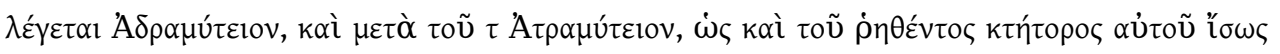

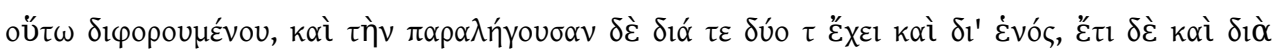

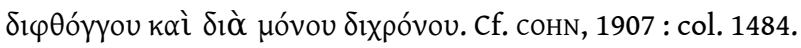

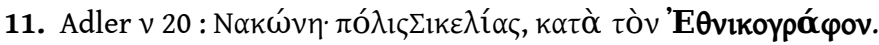

12. s.v. Anaktorion. Cf. HONIGMANN, 1929 : col. 2369. Voir FRASER, 2009 : 286-287.

13. s.v. Theoupolis et Sukai. Cf. HONIGMANN, 1929 : col. 2370 et 2371.

14. s.v. Darai. Cf.HONIGMANN, 1929 : col. 2371.

15. s.v. Akonai. Cf.honigmann, 1929 : col. 2370 ; PLRE, 3B, Petrus 6. HUNGER, 1978a : 300-303.

16. HONIGMANN, 1929 : col. 2372-2374 et juste avant lui MÜLLER, $1918: 340$ sq. Voir récemment la récapitulation des arguments par C. Neri (2008) à la suite de la publication de BILLERBECK et alii, 2006.

17. D'après P. Maraval dans le Dictionnaire des philosophes antiques (D 143), identification reprise par l'édition de M. Billerbeck ( $\alpha 7)$.

18. Adler $\varepsilon$ 3048: «Hermolaos, grammairien de Constantinople ayant écrit l'épitomé des

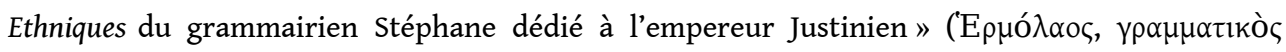

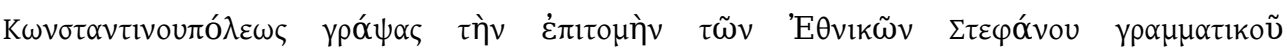

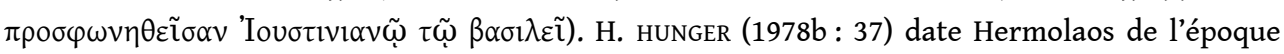
justinienne.

19. A l'article 'Høúxios, la Souda signale que cet auteur est la source de ce livre, sous-entendu pour les notices se trouvant dans l'Onomatologos. 
20. Il faut admettre l'ajout postérieur à Stéphane de Byzance d'une mention de Georgios Chœroboscos dans l'article Tamiathis comme le proposaient déjà A. MEINEKE (s.v.) et E. HONIGMANN (1929: col. 2371) ; M. Billerbeck ne conserve d'ailleurs pas ce témoignage parmi les éléments permettant de dater l'œuvre.

21. Adler к 342 : «Capiton, de Lycie, historien. Celui-ci a écrit des Isaurika en 8 livres, a traduit l'épitomé d'Eutrope, résumant en latin Livius le romain, et (écrit) un (ouvrage) Sur la Lycie et la

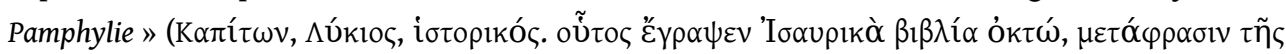

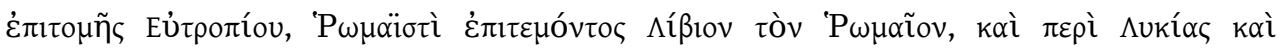
$\Pi \alpha \mu \varphi v \lambda i ́ \alpha \varsigma)$.

22. L'Isaurie est une région d'Asie Mineure au sud des monts du Taurus, encadrée par la Cilicie et la Pamphylie et qui correspond partiellement à l'ancienne Lycaonie.

23. On a lu à la suite de l'édition d'Aldus $\pi \varepsilon v \tau \varepsilon \kappa \alpha ı \delta \varepsilon \kappa \alpha ́ \tau \omega ~(15)$ pour le livre cité dans la notice Psimada. Meineke restitue d'après les manuscrits $\varepsilon^{\prime}$; il n'y a pas de raison de supposer plus de 8 livres à son œuvre.

24. On trouve le texte des fragments de cette œuvre dans l'édition de l'épitomé d'Eutrope (MGH SS aa 2, en bas du texte grec conservé de Paianios).

25. Sur Hesychios de Milet et son œuvre, voir en dernier lieu KALDELLIS, 2005.

26. On peut se demander s'il n'existe pas deux homonymes (un grand-père et son petit-fils? Un père et son fils ?), l'un plutôt de début $\mathrm{du} \mathrm{VI}^{\mathrm{e}}$ siècle et le second de la fin du règne de Justinien.

27. L'œuvre a probablement été résumée entre 827/829 et 857 ; au classement thématique (par genre littéraire) a été alors substitué un ordre alphabétique, plus commode à utiliser dans une période où l'on met en œuvre des lexiques.

28. On trouve très souvent l'indication erronée (y compris chez DEVREESSE, 1945) qu'il s'agit de son petit-fils.

29. La description codicologique est dans DEVREESSE, $1945: 208$.

30. Nous avons collationné, sur les clichés du manuscrit, le détail de toutes les variantes du manuscrit. Certaines n'apparaissent pas en effet dans l'édition de M. Billerbeck.

31. Le petit nombre d'annotations et la disparition d'une partie de la partie extérieure des annotations dans la marge ne permettent pas d'assurer une seule main mais cela semble vraisemblable.

32. Nous ne pouvons toutes les dénombrer ici ; voici quelques exemples, avec la référence à

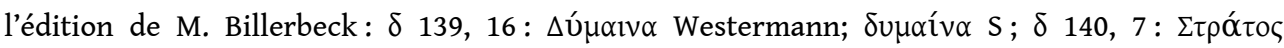

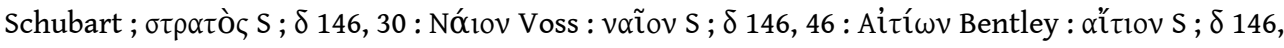

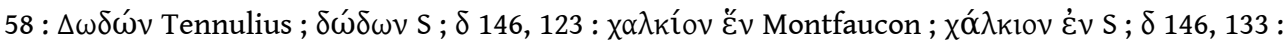

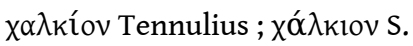

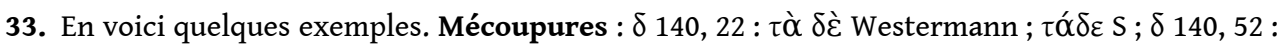

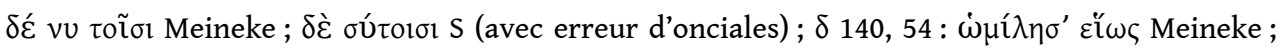

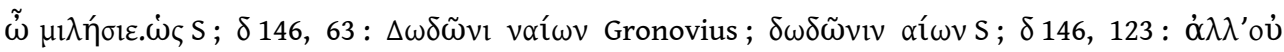

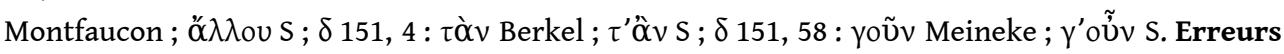

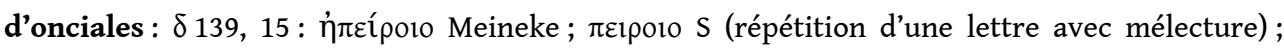
$\delta$ 140, 39 : $\Delta u \mu \alpha i ́ \alpha$ Berkel ; $\delta 1 \delta u \mu \alpha i ́ \alpha \mathrm{S}$ (répétition de 2 lettres avec mélecture et confusion avec le

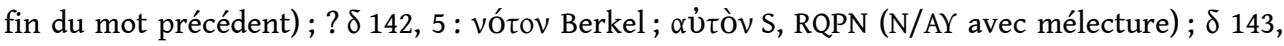

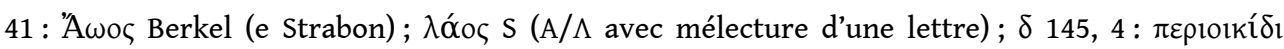

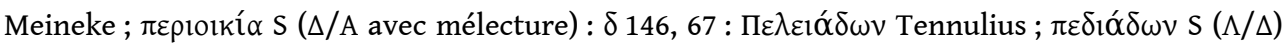
...

34. Sigle R: Wroclaw, Biblioteka Uniwersytecka, cod. graec. Rehdigeranus 47. Il s'agit d'un manuscrit de la fin $d u X V^{e}$ ou du début du XVI ${ }^{\mathrm{e}}$ siècle (176 folios, papier). La description précise est donnée par BILLERBECK et alii, 2006 : 9-10*. 
35. C'est pourquoi nous y reviendrons plus loin de façon plus détaillée, une fois que nous aurons décomposé les différentes phases de réduction de l'œuvre. Dans notre travail de thèse nous avons comparé finement les textes pour arriver à des certitudes.

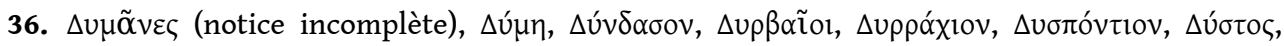
$\Delta \omega \delta \omega \dot{v \eta}, \Delta \omega v \varepsilon \tau \tau \tilde{\imath} v o l, \Delta \tilde{\omega} \rho \alpha, \Delta \omega ́ p 10 v, \Delta \tilde{\omega} \rho \circ \varsigma, \Delta \omega ́ \tau$ เov et "E $\alpha \rho \varepsilon \varsigma$ (notice complète ?).

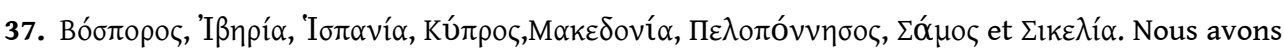
également étudié la notice $\Delta$ uppáxıov, qui fait partie de celles qui ont été conservées dans le manuscrit Coislinianus 228, afin de pouvoir comparer les changements opérés par Constantin Porphyrogénète.

38. La notice est largement incomplète du fait d'une altération du manuscrit. Le nombre de caractères de Stéphane de Byzance était donc beaucoup plus important à l'origine, ainsi que le nombre de citations.

39. Il s'agit du nombre de caractères, espaces non compris, d'après le texte du TLG (qui reprend l'édition de Meineke). Il peut exister une petite différence avec le nombre réel de signes (en particulier du fait de crochets ou autres signes d'édition) mais elle est minime et ne change pas les résultats de nos calculs.

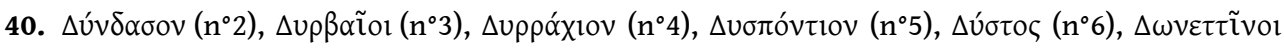

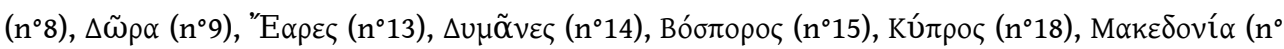

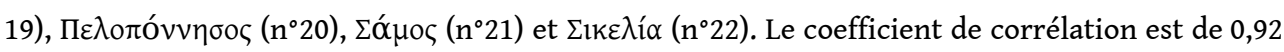
$(0,96$ si l'on enlève les notices de Constantin Porphyrogénète).

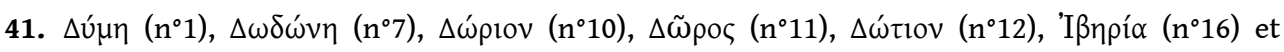
'I $\sigma \pi \alpha v i ́ \alpha\left(n^{\circ} 17\right)$. Le coefficient de corrélation est de 0,93.

42. Écrivain de langue grecque de date inconnue ( ${ }^{\mathrm{er}} \mathrm{s}$. ap. J.-C. ?) ayant rédigé une histoire des Phéniciens et une autre du Péloponnèse. La citation correspond à FgrHist $788 \mathrm{~F} 2$.

43. La moyenne des facteurs de réduction donne environ 5,6 pour la seule partie fiable de la fin de la lettre $\Delta$, soit $17,92 \%$ de conservation du texte.

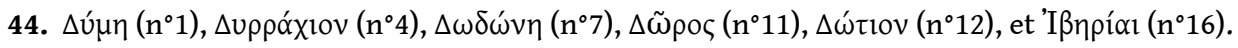

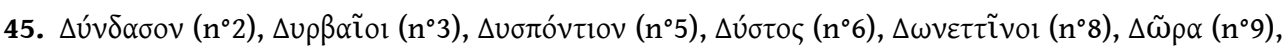

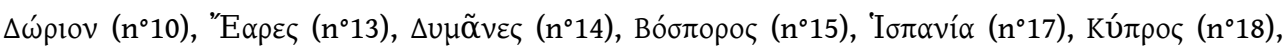

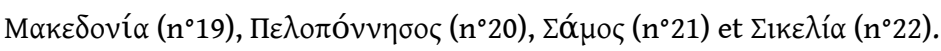

46. Seule la notice Bóoropoৎ a conservé 3 citations.

47. Nous ne parlons ici que de la citation d'un auteur, pas de la longueur elle-même. Compte tenu de la faiblesse de notre corpus comparatif, nous n'avons pas distingué, dans notre réflexion sur l'Épitomé, la seule mention d'un auteur de la citation véritable; cette dernière est de loin la moins fréquente dans le texte que nous avons conservé. Il n'est pas le lieu ici de détailler la question des citations.

48. Ce dernier a essayé d'aborder le problème de la réduction en appliquant quatre "schema" (selon le mot allemand utilisé par HoNIGMANN, 1929 : col. 2375-2376). Il conclut que l'Epitomé est l'œuvre d'au moins deux épitomateurs.

49. Le Rehdigeranus 47 (R), le Vaticanus Pal.gr. 253 (Q), le Vaticanus Pal.gr. 57 (P) et le Neapolitanus III.AA.18 (N). A. Meineke, qui ne connaissait pas tous les manuscrits utilisés par M. Billerbeck, utilise également pour les premiers livres le Perusinus 67 (П) mais celui-ci dérive du Vaticanus Pal.gr. 57.

50. Ce nombre résulte à la fois d'une mauvaise lecture de Tennulius et Montfaucon, rectifiée par

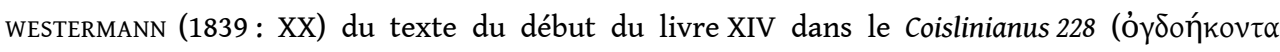

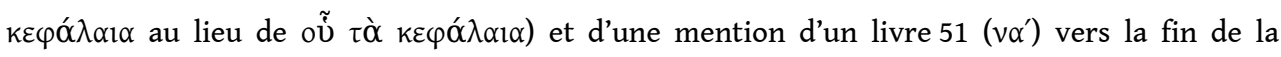
lettre $\sigma$.

51. Sur la restitution des livres, $c f$. HONIGMANN, 1929 : col. 2377-2379. 
52. Dans les deux tableaux suivants, les numéros des livres dont la délimitation est donnée dans les manuscrits sont notés en gras dans la colonne de gauche.

53. Nous n'avons pas comptabilisé le texte de Constantin Porphyrogénète pour la notice de Bóбтороৎ.

54. Les 2220 caractères des notices communes de l'Épitomé avec les fragments de Stéphane de Byzance correspondent à $23 \%$ du total du livre XIII et à 13150 caractères dans l'œuvre originale. Nous ne possédons donc qu'un quart d'un livre de Stéphane de Byzance.

55. Nous n'avons pas comptabilisé le texte de Stéphane de Byzance pour la notice "E $\alpha \rho \varepsilon \varsigma$.

56. On l'a dit, les notices sont de taille très diverse suivant le nombre de formes lexicales et donc de citations. De plus, l'Épitomé ne rend pas compte de la taille des notices d'origine.

57. Dans les codices, la longueur du livre importe peu, contrairement au volumen des époques antérieures.

58. Nous avons peu de repères pour les derniers volumes. Nous proposons de corriger la mention de livre LII ( $\left.\vee \beta^{\prime}\right)$ en livre LIV $\left(\nu \delta^{\prime}\right)$ avant T $\lambda \tilde{\eta} \tau \eta \varsigma$ car le livre LII correspondrait à celui qui débute avec le lemme Tó $\beta \alpha$.

59. Cette observation avait déjà été énoncée mais n'a jamais fait l'objet d'une étude plus approfondie : «It also seems probable that the work, as it now exists, is not a fair representation of the epitome of Hermolaus, but that it has been still further abridged by successive copyists. The former part of the work is pretty full ; the portions from Пó $\tau \rho \alpha$ to the middle of $\Sigma$ is little more than a list of names; the articles in $T$ and $Y$ become fuller again; and those from $X$ to $\Omega$ appear to be copied, almost without abridgment, from the work of Stephanus. » (Smith 1844-1849, 3, col. 906).

60. Ce doublon, déjà signalé par Meineke, a servi à HONIGMANN (1929 : col. 2377-2379) à proposer un deuxième épitomé mais qu'il ne définit pas. Repris par FRASER, 2009 : 288.

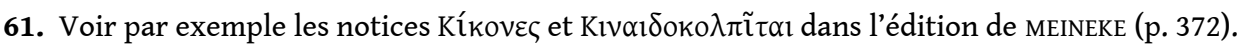

62. Nous appliquons le taux moyen de réduction de 5,58, corrigé par le taux de réduction mis en évidence pour chaque livre. Nous insistons sur le fait qu'il s'agit ici de donner une idée de la taille d'un livre mais que ce calcul reste avant tout théorique.

63. Arrondi au feuillet près. Le Perusinus 67 est un manuscrit de la fin du XV $\mathrm{X}^{\mathrm{e}} \mathrm{s} .(280 \times 205 \mathrm{~mm})$ qui comprend 173 feuillets (soit 346 pages). La première partie, rédigée par un scribe anonyme, comporte environ une cinquantaine de caractères par ligne et une trentaine de lignes par page ( HOFFMANN, 1983: 120-122 avec reproduction photographique p. 121), soit une moyenne de 1500 signes par page.

64. Sur la translittération et les conséquences sur les manuscrits, voir entre autres IRIGOIN, 2001 : 85-87 et RONCONI, 2003.

65. La même constatation semble pouvoir être faite pour la Collection d'hippiatrie grecque, recueil de textes plus anciens concernant les chevaux (Doyen-Higuet 2006, p. 112-113) et les textes médicaux (MARGANNE, 2004 : 127-131). Voir également WILSON, 1988 sur le passage du volumen au codex, et ses conséquences.

66. Sur l'onciale, voir l'étude très classique de G. CAVALLo (1967).

67. Nous prendrons comme exemple d'un manuscrit du vi siècle le Codex Guelferbytanus $A$, qui contient le texte du Nouveau Testament sur deux colonnes de 24 lignes, avec une moyenne de 12 caractères par ligne et par colonne.

68. La datation tardive est établie depuis quelques décennies seulement, mais elle est maintenant certaine du fait de la mise en évidence de citations de Jean Damascène. Voir les références bibliographiques données par E. DICKEY (2007: 80).

69. Prolegomena et scholia in Theodosii Alexandrini canones isagogicos de flexione verborum, p. 305 de l'édition d'A. HILGARD (1894).

70. Id., p. 42. 
71. Notre propos ici n'est pas de retrouver la totalité des utilisations de Stéphane de Byzance mais simplement de montrer la transmission intellectuelle de son œuvre. M. Billerbeck a noté dans son apparat critique les références pouvant se rapporter à cet auteur.

72. Cod. 145 à 153. Cod. 145 Helladius, Lexique des mots et des phrases usités en prose; cod. 146 (anonyme), Lexique pour le 'style classique'; cod. 147 (anonyme), Lexique pour le 'style solennel' ; cod .148 (anonyme), Lexique pour le 'style politique'; cod. 149 Valérius Pollion, Lexicon; cod. 150 Julien, Lexique; Philostrate, Lexique; Valérius Diodore, Lexique ; cod. 151 Timée, Lexique pour Platon; cod .152 Denys d'Halicarnasse, Lexique des mots attiques; cod. 153 Pausanias, Lexique.

73. Aimon, l'airain de Dôdônê, Ilissos, Messapion.

74. Sur la question de la datation des deux livres et la probable rédaction du livre II dans le cadre du cercle encyclopédique voulu par Constantin, voir PERTUSI, 1952 : p. 39 sq. Paul LEMERLE (1971: 279) considère pour sa part que la totalité de l'œuvre a été rédigée par Constantin. Pertusi pense que le livre II est postérieur, de la fin $\mathrm{du} \mathrm{x}^{\mathrm{e}}$ siècle ; il nous semble toutefois contradictoire d'avoir des mentions de Stéphane de Byzance dans un livre daté de cette époque et de constater que la Souda, à la même date, n'utilise pas son œuvre pourtant importante d'un point de vue lexicographique. T. C. Lounghis (1973) propose de manière convaincante l'intervalle de date 952-957 pour la rédaction de ce livre. En revanche, il ne distingue pas les deux livres et considère qu'il s'agit d'une œuvre homogène, ce qui n'est probablement pas le cas.

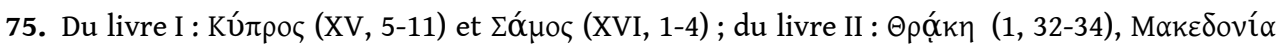

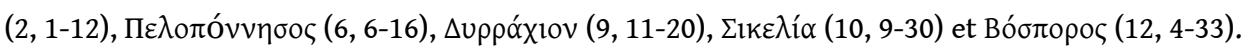

76. La datation est donnée par BURY, 1906 et JENKINS \& MORAVCISK, 1967. Sur l'œuvre, voir également SODE, 1994.

77. § 23 : 'I $\sigma \pi \alpha v i ́ \alpha ; \S 24$ : 'I $\beta \eta \rho i ́ \alpha$.

78. Les Excerpta Constantiniens, dont ne subsistent plus que cinq recueils encore souvent incomplets, regroupaient de façon thématique des extraits des d'historiens entre le $v^{\mathrm{e}}$ siècle av. J.-C. et le VII ${ }^{\mathrm{e}}$ siècle de notre ère.

79. Une œuvre de type « lexique » n'entrait logiquement pas dans le projet des Excerpta.

80. DILLER, $1950: 241-253$, repris par M. BILLERBECK et alii, $2006: 29-30^{*}$.

81. Sur ces questions de mouvements de recueils dans l'entourage de Constantin, voir odorico, 2011.

82. Ces annotations proviennent à l'origine du manuscrit Marcianus 448, version conservée de la Souda de la main même d'Eustathe de Thessalonique.

83. Nous insistons sur le fait qu'il s'agit simplement, par ce calcul, d'avoir un ordre d'idée de l'ampleur du manuscrit du $\mathrm{XI}^{\mathrm{e}}$ siècle.

84. Le fragment conservé est un quaternion (donc 8 folios); c'est probablement le format standard du manuscrit.

85. Nous n'en avons d'ailleurs aucune trace dans les manuscrits conservés.

86. Avec le calcul réalisé ci-dessus, le manuscrit [ $\beta]$ aurait pu contenir un peu plus de 800 folios.

87. On a déjà mis en évidence le fait qu'Eustathe et les étymologistes ont utilisé les mêmes sources (donc probablement fréquenté les mêmes bibliothèques) : coHN, 1907 : col. 1475.

88. Les mentions sont rassemblées dans l'apparat critique de l'édition de M. Billerbeck.

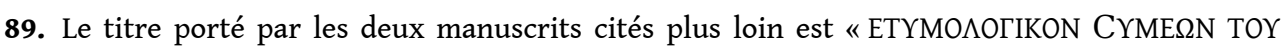
МЕГА $О Y$ ГРАММАТIKOY »; cf. REITZENSTEIN, $1897: 254$.

90. G. BERGER (1972: XVII-XVIII) a montré que l'Etymologicum Symeonis a servi de source à l' Etymologicum Magnum et plus tard au lexique du pseudo-Zonaras. Voir également SCHIRONI, 2004 : 18.

91. Découverte seulement au XIX ${ }^{\mathrm{e}}$ siècle, cette compilation est conservée sous une forme abrégée dans deux manuscrits du $\mathrm{x}^{\mathrm{e}}$ siècle, présentant des versions différentes, ce qui explique qu'elle n'ait jamais été éditée de façon exhaustive. Deux projets éditoriaux distincts, l'un sous la 
direction de Klaus Alpers et le second par François Lasserre et Nikolaos Livadaras, ont publié le début du lexique.

92. Cet auteur nous est actuellement totalement inconnu. Il n'est cité que par cet ouvrage et pourrait correspondre au patriarche de la première moitié $\mathrm{du} \mathrm{IX}^{\mathrm{e}}$ siècle. Il faut noter que R. Reitzenstein (1897), dans son étude des sources de l'Etymologicum Genuinum, le cite sans jamais donner aucune précision sur lui.

93. Voir par exemple Althaia, Alônis, ...

94. Contrairement aux autres lexiques, celui-ci a été intégralement publié, en particulier par Thomas GAISFORD (1848).

95. Dans son apparat critique elle rattache toutefois ces mentions à l'Etymologicum Symeonis.

96. Sur Eustathe et ses commentaires sur Homère, voir PONTANI, 2005 : 173-178.

97. Références dans BILLERBECK et alii, $2006: 34^{*}$ et n. 59. Cf. HUNGER, 1978a : 510.

98. Là encore notre recherche sur ces aspects ne vise pas l'exhaustivité mais un ensemble suffisamment représentatif pour comprendre les transformations de l'œuvre.

99. Nous reprenons ici plus en détail les observations qui auraient pu trouver leur place supra, $\S 2.1 .1$.

100. C'est moins vrai pour la notice ôlenos, dont on a deux mentions distinctes. Eustathe semble l'avoir utilisée de façon très sélective et presque incorrecte.

101. A. DILLER (1938: 335) suit la démonstration de W. KNAUSS (1910).

102. Georgios Choeroboscos, Eis ton onomatikon, $54 \mathrm{~b}$. Les autres villes signalées ici, ville

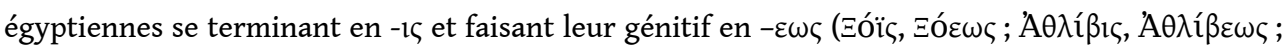

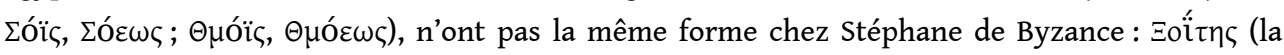

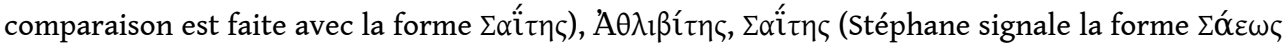

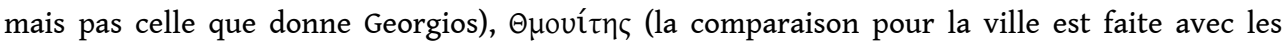

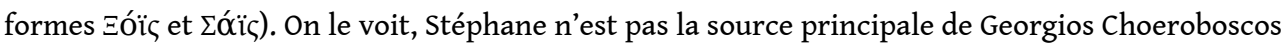
pour ces formes.

103. Sur ce lexique, voir ci-après $\S 3.4 .1$.

104. Lettre chi, p. 1858.

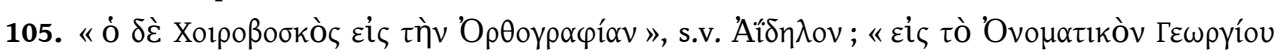

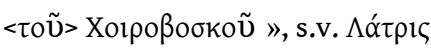

106. A. DILLER (1938 : 334, n. 3) propose en effet que l'interpolation provienne d'Eustathe.

107. Sur le contexte général de la préservation des textes à ce moment, voir REYNOLDS, WILSON, 1991.

108. D'après F. LASSERRE (1959 : 45-46), Nicéphore Grégoras (v. 1295-1360), surtout connu pour ses œuvres astronomiques, a recopié des excerpta de Strabon et probablement la mention que nous signalons ici, qui est donc plus ancienne.

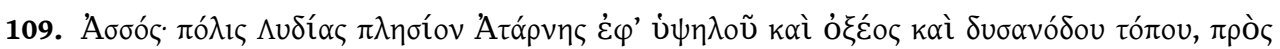

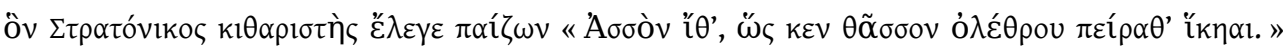

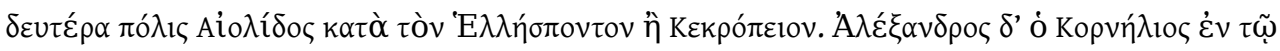

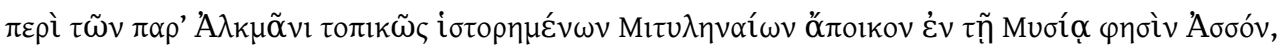

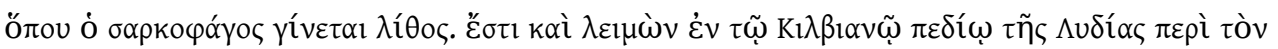

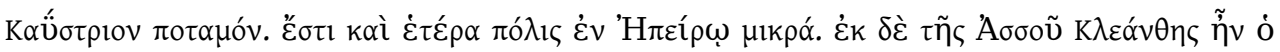

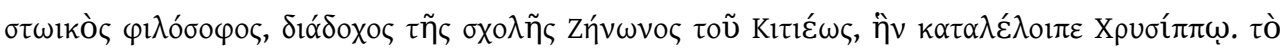

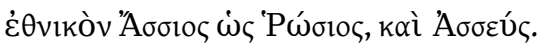

110. Sur ce manuscrit, voir OHME, $1990: 103$.

111. L'indication est dans DILLER, 1975 : 94.

112. La transcription est de M. Billerbeck.

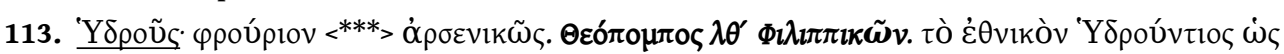

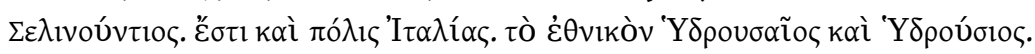




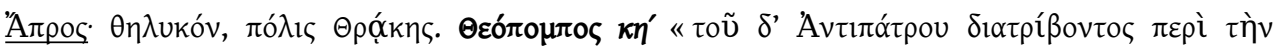

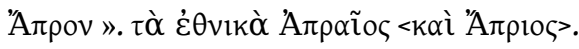

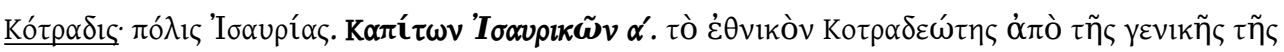

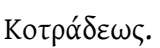

114. La datation est variable suivant les auteurs. M. BILLERBECK et alii $\left(2006: 31^{*}\right)$ indique le XIII ${ }^{\mathrm{e}}$ siècle. A. ACCONCIA LONGO et A. JACOB (1983) le datent du XIV ${ }^{\mathrm{e}}$ siècle. Nous préférons la datation de ces derniers qui repose sur une meilleure connaissance des manuscrits otrantais.

115. L'édition actuelle de l'Etymologicum Gudianum remonte au XIX ${ }^{\mathrm{e}}$ siècle (STURZ, 1818).

116. Sur tout ceci, voir la mise au point récapitulative de F. SCHIRONI (2004:16-17 et la n. 13 pour l'Etymologicum Casulanum).

117. Il n'est pas certain que le livre XIV soit le résumé du second abrégé. Cela semble toutefois possible en fonction du pourcentage de réduction.

118. Voir en dernier lieu le recensement des manuscrits par M. BILLERBECK et alii (2006: $\left.7^{*}-29^{*}\right)$, comme A. DILLER (1938) avant elle, qui conclut à un archétype commun connu aux environs de 1480 et les remarques de C. NERI (2008).

119. C'est entre 1492 et l'editio princeps d'Aldus Manutius de 1502 que 17 manuscrits ont été copiés (MARCOTTE, $1987: 193$ ).

120. On trouvera dans mARCotTE (1987: 192 sq.) la transcription de l'acte notarié de l'inventaire de 1503 (le manuscrit de l'abrégé est le n¹0).

121. L'editio princeps comporte une dédicace à Giovanni Taberio datée du XV des Calendes d'avril 1502 (18 mars).

\section{RÉSUMÉS}

Le lexique géographique de Stéphane de Byzance, appelé sous une forme abrégée Ethnika, rassemble les "géonymes" ayant une forme dérivée, suivant la définition des grammairiens antiques (ethnique, féminin, ktétique...). Il fait l'objet ici d'une relecture attentive, en analysant en particulier les taux de réduction variables suivant les parties de l'œuvre (divisée probablement à l'origine en 60 livres). Il nous est en effet parvenu sous une forme abrégée, attribuée, d'après la Souda, à Hermolaos ( $\mathrm{VI}^{\mathrm{e}}$ siècle ?). Le texte que l'on connaît est en fait issu d'un manuscrit complet $\mathrm{du} \mathrm{XI}^{\mathrm{e}}$ siècle, translittéré d'un manuscrit en onciales pouvant remonter à Stéphane de Byzance. Un premier abrégé intervient probablement dans la première moitié du XII e siècle; c'est celui qu'utilise Eustathe de Thessalonique. Par la suite, l'œuvre est à nouveau abrégée à deux reprises jusqu'à la constitution de l'archétype (disparu) des manuscrits que nous connaissons, peut-être dans le contexte de la région d'Otrante $a u \mathrm{xv}^{\mathrm{e}}$ siècle. La connaissance de l'histoire complexe de ce texte est un préalable pour une utilisation rationnelle des informations qu'il contient puisqu'il est bien souvent le seul à nous transmettre des mentions de certains auteurs de langue grecque.

Stephanus of Byzantium's geographical lexicon, called Ethnika in an abbreviated form, gathers the "geonymes" which have a derived form, as defined by the ancient grammarians (ethnic, female, ktetic...). It's been subject here of a careful reading, analyzing in particular the variable rates of reduction according to the parts of the work (probably originally divided into 60 books). We have it in an abbreviated form, attributed, according to Souda, to Hermolaos (sixth century?). 
In fact, the text that we know comes from a complete manuscript of the eleventh century, transliterated from an uncial manuscript which can go back to Stephanus of Byzantium. A first abridgment probably involved in the first half of the twelfth century, and is the one used by Eustathius of Thessalonica. Subsequently, the work was again shortened twice untill the constitution of the archetype (missing) of all the manuscripts we know, perhaps in the context of Otranto in the fifteenth century. Knowledge of the complex history of this text is a prerequisite for a rational use of the information it contains, since it is often the only to give us some references to Greek authors.

\section{INDEX}

Keywords : ancient geography, epitome, Stephanus of Byzantium, condensed text, Hermolaos Mots-clés : géographie antique, épitomé, Stéphane de Byzance, abrégé, Hermolaos

\section{AUTEUR}

\section{MARC BOUIRON}

marc.bouiron@ville-nice.fr.

Conservateur du Patrimoine et directeur du service archéologique de la Ville de Nice. Spécialiste d'archéologie urbaine, ses travaux ont porté principalement sur Marseille et Nice à l'époque médiévale et moderne (conservateur de la crypte archéologique de Nice et directeur des fouilles de la colline du Château). Ses travaux universitaires précédents, sur l'habitat antique de Glanum, l'avaient amené à côtoyer le texte de Stéphane de Byzance. L'élargissement d'une recherche spécifique sur les notices de cet auteur concernant le Midi de la Gaule puis l'Europe occidentale a entraîné une révision totale du texte et des sources de ce compilateur antique, dans le cadre d'un doctorat en cours à l'Université de Nice (CEPAM). 